\title{
A NÃO LINEARIDADE DO NEXUS DESENVOLVIMENTO FINANCEIRO - CRESCIMENTO ECONÔMICO: O CASO DOS PAÍSES PRODUTORES DE PETRÓLEO
}

\author{
THE NONLINEARITY OF NEXUS FINANCIAL DEEP - ECONOMIC GROWTH: THE \\ CASE OF OIL PRODUCING COUNTRIES
}

\begin{abstract}
José Alberto Fuinhas Antonio Cardoso Marques

RESUMO Rúben Simão

O presente estudo apresenta uma nova perspectiva da influência do Desenvolvimento Financeiro sobre o Crescimento Econômico num painel de 15 países produtores de petróleo, com um período temporal de 1981 a 2011. Incorporou-se no modelo variáveis como as rendas de petróleo, o rácio de produção de petróleo sobre consumo de energia primária, preços de petróleo, exportações de bens e serviços, consumo de petróleo e emissões de $\mathrm{CO} 2$. Utilizou-se o modelo ARDL, para analisar as relações dinâmicas entre as variáveis no longo e no curto prazo. Seguidamente utilizou-se o estimador Driscoll-Kray, com efeitos fixos, para corrigir a significância dos parâmetros estimados. Os resultados demonstram a não linearidade no longo prazo, através do Teste U, entre Desenvolvimento Financeiro e Crescimento Econômico nos estudos formulados. Devido ao longo período estudado, às variáveis incluídas e ao uso de técnicas econométricas adequadas, ampliou-se a literatura do nexus Desenvolvimento Financeiro-Crescimento Econômico.
\end{abstract}

Palavras-chave: Desenvolvimento financeiro, crescimento econômico, não linearidade.

\begin{abstract}
The influence of Financial Development on Economic Growth was assessed with a panel of 15 countries oil producers, for the period between 1981 and 2011 . Moreover, variables like oil rents, the ratio of oil production to primary energy consumption, oil prices, exports of goods and services, oil consumption and $\mathrm{CO} 2$ emissions were used. The ARDL model come out as the most suitable for estimation. Indeed, this model consents the analysis of the dynamic relationships between short and long term variables. To refine the significance of the estimated parameters and introduce robustness to the archived findings, the Driscoll-Kray estimator was used with fixed effects. The nonlinearity between Financial Development and Economic Growth was considered and confirmed at long term, with U Test. Due to the incorporated variables and the econometric techniques, the obtained results come out as appropriated and useful to fill some gaps in the literature.
\end{abstract}

Keywords: Financial development, economic growth, nonlinearity

JEL: C33, O11, O57, Q43

\footnotetext{
${ }^{1}$ Doutor em Economia pela Universidade da Beira Interior (UBI-Portugal). Professor da Universidade da Beira Interior (UBI-Portugal). E-mail: fuinhas@ubi.pt
} 


\section{Introdução}

As interações entre Crescimento Econômico (CE), produção de petróleo, emissões de $\mathrm{CO}_{2}$ e Desenvolvimento Financeiro (DF) têm sido alvo de diversos estudos científicos ao longo das últimas décadas. Entender os determinantes destas relações torna-se assim importante para perceber os mecanismos envolventes.

O consumo e produção de petróleo continua a desempenhar um papel relevante na literatura atual, não apenas com o objetivo de perceber o seu papel no $\mathrm{CE}$, bem como a incorporação de outros fatores exógenos (como, por exemplo, as emissões de $\mathrm{CO}_{2}, \mathrm{o}$ preço do petróleo e as variáveis financeiras) presentes na economia mundial e que influenciam o nexus crescimento-energia. Após Kraft e Kraft (1978) o estudo no âmbito da economia energética aumentou significativamente, visto este apenas abordar as vertentes consumo de energia e Produto Interno Bruto (PIB), tornando assim os resultados pouco influentes em comparação com os mais recentes. Com o começo das investigações científicas relacionadas com o nexus crescimento-energia, surge a preocupação com as questões ambientais, nomeadamente a poluição e as emissões de $\mathrm{CO}_{2}$. A literatura demonstra que existe uma relação positiva, estatisticamente significante, entre as emissões de $\mathrm{CO}_{2}$ e o consumo de energia (Saidi e Hammami, 2015).

Outra componente da economia mundial e energética que se deve ter em conta é o Desenvolvimento Financeiro. Este determinante é bastante controverso no histórico da literatura económica e energética. Segundo alguns autores, o DF indica a quantidade real de dinheiro para ser utilizado em projetos de investimento (Goldsmith, 1969; Minier, 2009; Sadorsky, 2010). Diversos autores afirmam que o DF tem uma forte e positiva ligação com o CE (Schumpeter e Opie, 1934; Gurley e Shaw, 1955). Na

opinião contrária surgem outros autores que advertem empiricamente, para que o desenvolvimento do setor financeiro não seja visto como um fator apenas vantajoso para a economia (Kaminsky e Reinhart, 1999; Deidda e Fattouh, 2002; Wachtel, 2003).

Assim, o estudo realizado analisa variáveis de teor energético, como produção, consumo e preços de petróleo, emissões de $\mathrm{CO}_{2}$ e uma variável financeira, sendo ela uma proxy para o medir o Desenvolvimento Financeiro. Procuramos entender qual a relação entre Desenvolvimento Financeiro, Crescimento Econômico e consumo de energia nos países produtores de petróleo. 
O estudo realizado tem por análise um horizonte temporal de 31 anos (19812011), para um total de 15 países produtores de petróleo. Os países utilizados representam uma amostra diversificada dos países produtores de petróleo, dado que inclui países com diferentes níveis de produção de petróleo. Utiliza-se o modelo Autoregressive Distributed Lag $^{2}$ (ARDL), dado que o horizonte temporal é longo e é esperado que existam relações dinâmicas entre as variáveis em investigação.

O artigo está estruturado da seguinte forma: secção 1, "Revisão da Literatura", apresenta uma visão global das investigações teóricas/empíricas já realizadas no campo da interação entre CE e DF; secção 2, "Dados e Metodologia", expõe os dados sobre os quais vai incidir este trabalho e as abordagens/metodologias utilizadas; secção 3, "Resultados", apresenta os resultados da análise empírica; secção 4, "Discussão", relata as evidências empíricas retiradas dos resultados e por fim com a "Conclusão", encerramos o estudo com a apresentação de algumas considerações.

\section{REVISÃO DE LITERATURA}

A relação entre DF e CE é um dos temas da economia global mais debatidos na literatura teórica e empírica. Deste debate resultou um conjunto de fatos estilizados. Segundo Graff (2002), os trabalhos já realizados e que estudam este tema podem ser agrupados em quatro linhas orientadoras: (i) não há uma relação entre CE e DF; (ii) o DF decorre do CE; (iii) o DF é importante no CE, proporcionando um melhor monitoramento da qualidade do investimento (Goldsmith, 1969); (iv) o CE pode ser fragilizado pelo desenvolvimento do setor financeiro, devido às crises que o setor financeiro sofre periodicamente (Keynes, 1988; Krugman, 1996).

Os primeiros estudos que abordam estas questões remontam à década de 40 , em que Schumpeter e Opie (1934) destacaram o papel das instituições financeiras na inovação e investimentos produtivos. Por exemplo, Gurley e Shaw (1955), Mckinnon (1973) e Shaw (1973) aprimoraram o estudo de Schumpeter e Opie (1934).

Alguns destes artigos, nomeadamente Gurley e Shaw (1955) argumentaram que os mercados financeiros mais desenvolvidos promoviam o $\mathrm{CE}$, isto através da mobilização da poupança para investimentos produtivos. Este pensamento é analisado mais tarde por $\mathrm{Xu}$ (2000) que encontra na sua pesquisa fatos importantes para o DF, argumentando que a realização de investimento afetaria positivamente o CE.

\footnotetext{
2 São escritos termos técnicos em inglês para não haver distorção do sentido das palavras caso fosse realizada uma tradução para português.
} 
Demetriades e Hussein (1996) realçaram que o efeito de desenvolvimento do setor financeiro promovia o $\mathrm{CE}$ em economias mais desenvolvidas.

Diversos estudos exploraram o DF e o CE, utilizando uma abordagem baseada no estimador Ordinary Least Squares (OLS), concluindo que a relação entre eles seria positiva (Golsmith, 1969; King e Levine, 1993a, 1993b; Levine e Zervos, 1998). Por outro lado, existem outros autores que argumentam que a abordagem referida pelos autores anteriores não seria a mais correta, criticando a utilização do estimador OLS. O setor financeiro deve ser analisado como fenômeno de longo prazo, devido ao fato de que as conclusões retiradas por esses autores, com base em análises cross-country, não seriam viáveis e apresentariam problemas econométricos (Barro, 1991; Khan e Senhadji, 2003; Chuah e Thai, 2004). A falta de dados disponíveis não permite melhorar, econometricamente, os estudos sobre o DF, sendo necessário utilizar séries temporais para aprimorar empiricamente os artigos (Christopoulos e Tsionas, 2004; Beck, 2008). Porém, a utilização de dados em painel, permite reduzir as deficiências colocadas por séries temporais e de crosses secionais (Calderon e Liu, 2003; Dawson, 2010).

Loayza e Ranciere (2006) empregaram no seu estudo uma amostra de 75 países, com um horizonte temporal de 41 anos (1960-2000), servindo-se do estimador Pooled Mean Group (PMG), este método permite a heterogeneidade dos parâmetros e a separação dos efeitos no curto e longo prazo. Encontraram assim uma interação positiva e significante entre CE e DF no longo-prazo, enquanto no curto prazo não se estabelece resultados significantes. Este estudo concentrou-se apenas na relação linear entre as variáveis. Jedidia et al. (2014) mostraram no seu estudo que o crédito interno ao setor privado influenciava positivamente o $\mathrm{CE}$, sendo que no longo prazo o DF é mais influente sobre o PIB, do que no curto-prazo.

Utilizando um período temporal de 1960 a 2001 e testes de cointegração e causalidade, Ang e Mckibbin (2007) constataram que a liberalização financeira estimula o DF. Apenas com a remoção de políticas restritivas (repressionist policies) é que o CE e DF estão positivamente relacionados.

O DF e CE estão relacionados com produção de bens e serviços e consequente consumo de energia. Surge então outro determinante relevante, a energia. O primeiro estudo que analisava a economia da energia foi efetuado por Kraft e Kraft (1978), no contexto da concepção de políticas produtivas de conservação de energia. Desde este estudo que o consumo de energia e CE têm sido alvos de diversas e diferentes 
abordagens/metodologias. Perante a literatura existente, pode-se resumir o nexus crescimento-energia em 4 hipóteses postuladas: conservação, crescimento, feedback e neutralidade (e.g. Narayan e Smith, 2008; Apergis e Payne, 2009; Zhang e Cheng, 2009; Wolde-Rufael, 2009; Ozturk, 2010).

A incorporação de proxies para medir o DF, corresponde a um aperfeiçoamento na análise do nexus crescimento-energia. Analisando um painel de 58 países, entre 1990 e 2012 e utilizando o modelo dinâmico e o estimador em painel Generalized Method of Moments (GMM), a evidência empírica releva que há um impacto significativo entre emissões de $\mathrm{CO}_{2}$ e consumo de energia, e que o DF, medido através do crédito privado em percentagem do PIB, apresenta significância estatística positiva no consumo de energia no painel global e em continentes específicos, como Europa, Ásia e América Latina (Saidi e Hammani, 2015). Na Europa, nomeadamente nos membros da União Europeia (UE) mais antigos (15 países), entre 1990 e 2011, encontra-se um forte impacto do DF no consumo de energia, independentemente do desenvolvimento ocorrer no setor financeiro ou no mercado de ações. Com a junção dos novos membros na UE (27 países no total) o impacto do DF varia consoante a medida usada no modelo, evidenciando a presença de um $U$ invertido ou nenhuma relação estatisticamente significante. Este estudo utiliza o modelo GMM como estimador dos resultados (Çoban e Topcu, 2013).

Outro estudo, com o modelo GMM também em vigor, relaciona o impacto do desenvolvimento do setor financeiro no consumo de energia numa amostra de 9 países da Europa Central e do Leste, entre 1996-2006. Os resultados empíricos demonstram uma relação positiva e significante entre DF e consumo de energia, quando a variável de desenvolvimento é mensurada por "deposit money bank assets to GDP, financial system deposits to GDP, or liquid liabilities to GDP” (Sadorsky, 2011). Resultados similares foram encontrados numa amostra para 19 países entre 1980 e 2008 (Al-Mulali e Sab, 2012). Fortes indícios de bidirecionalidade entre CE e DF são relatados numa amostra de 109 países (de 1960 a 1994) por Calderon e Liu (2003), esta causalidade foi também abordada por Luintel e Khan (1999).

A investigação entre o setor financeiro, CE e energia apresenta uma literatura bastante diversa e extensa. A preocupação com o meio ambiente é um dos fatores incluídos na literatura do nexus crescimento-desenvolvimento-energia. Alguns estudos efetuados tiveram em consideração o DF como um determinante relevante no desempenho ambiental, argumentando que um elevado DF promove projetos de baixo 
custo para o ambiente (Grossman e Krueger, 1995; Claessens e Feijen, 2007; Tamazian et al., 2009; Tamazian e Bhaskara Rao, 2010). Algumas razões para a inclusão no estudo do desenvolvimento do setor financeiro na discussão entre CE e ambiente são: (i) o incentivo à investigação e pesquisa, por parte do investimento direto estrangeiro (IDE) de forma a afetar a dinâmica da performance ambiental (Frankel e Romer, 1999); (ii) a motivação e oportunidade de utilizar tecnologia limpa e sofisticada (Birdsall e Wheeler, 1993; Frankel e Rose, 2002); (iii) a oportunidade de CE, originando maior produção e consequente geração de poluição (Jensen, 1996; World Bank, 2000).

A pesquisa levada a cabo por Jalil e Feridun (2011) da economia chinesa no período de 1953 a 2006, recorrendo ao método ARDL, obteve como conclusão a existência de uma relação negativa e significante entre DF e poluição do meio ambiente, sendo o rendimento, consumo de energia e exportações os maiores responsáveis pela poluição nessa economia. Analisando os países BRIC, de 1992 a 2004, Tamazian et al. (2009) investigaram não só a causalidade do CE e ambiente, como incluíram o setor financeiro no estudo. Concluíram que um alto grau de CE e DF diminui a degradação do meio ambiente e que a adoção de políticas de liberalização do setor financeiro que impulsionam a Investigação e Desenvolvimento (I\&D) direcionadas para o investimento direto estrangeiro reduzem a poluição. Aplicaram na sua amostra de dados os países Japão e Estados Unidos da América, para determinar a robustez do modelo e as conclusões finais mantiveram-se. A não existência de uma conclusão definitiva relativamente ao nexus consumo de energia, PIB, DF e emissões de CO2, originaram a pesquisa de Ziaei (2015) através da utilização de Panel Vector Auto Regression (PVAR), em 13 países europeus e 12 do Este Asiático e Oceania, entre 1989 e 2011. Aferiu-se a importância da bidirecionalidade entre emissões de $\mathrm{CO} 2$ e consumo de energia e que a existência de choques no consumo de energia e emissões de $\mathrm{CO} 2$ não se pronuncia tanto nos indicadores financeiros como na taxa de retorno de ações, sendo que esta é mais sentida nos países europeus.

A diversidade existente na literatura relativa ao meio ambiente, utiliza múltiplas maneiras de expor o problema referente à performance ambiental. As emissões de $\mathrm{CO}_{2}$ são um dos maiores causadores da poluição e por esse fato são também um determinante muito estudado na comunidade científica. O tema entre DF e emissões de $\mathrm{CO}_{2}$ é uma questão pertinente, por exemplo, na Malásia através de uma análise de causalidade à Granger, verifica-se uma significante relação de longo prazo entre emissões de $\mathrm{CO}_{2}$, DF, consumo de energia e CE. Afere-se ainda que o DF reduz a 
emissão de $\mathrm{CO}_{2}$ para a atmosfera (Shahbaz et al., 2013). Na Índia os resultados e metodologia foram idênticos, analisou-se a relação de longo prazo entre emissões de $\mathrm{CO}_{2}$, DF, $\mathrm{CE}$, consumo de energia e o sumário de exportações mais importações (trade opennes) de 1971 a 2008 e concluiu-se que o DF reduz as emissões de $\mathrm{CO}_{2}$, mostrando a importância da geração de políticas energéticas na economia indiana para uma melhor preservação ambiental (Boutabba, 2014). Analisando 30 economias africanas, Al-Mulali e Sab (2012) averiguaram que o consumo de energia, e consequente emissão de $\mathrm{CO}_{2}$, tinha um papel importante no CE e no desenvolvimento do setor financeiro. Este estudo foi realizado para economias pouco desenvolvidas, e por isso a criação de um melhor setor financeiro gera poluição. Seria necessária a adoção de medidas no setor da energia, para estas economias conseguiram alcançar o potencial e eficiência energética.

Em economias produtoras de petróleo, a análise empírica sobre o nexus Crescimento-Desenvolvimento-Energia é notada na literatura. Utilizando dados anuais entre 1974 e 2008 com o método estimador ARDL, nos Emirados Árabes Unidos, os resultados declararam a existência de uma relação significante e negativa entre DF, medida pela proxy M2 (money and quasi-money), e o CE. Estes resultados demonstram que as instituições financeiras em economias exportadoras de petróleo são fracas (AlMalkawi et al., 2012). Outro estudo que examina economias produtoras de petróleo, utilizando o método GMM entre 1960 e 2001, demonstra que o DF é mais fraco em economias produtoras de petróleo do que importadoras. Os autores justificam este fato com o argumento de falta de eficiência das instituições financeiras em países dependentes das exportações de petróleo (Nili e Rastad, 2007). Separando o setor petrolífero do setor não petrolífero para perceber como o DF influência o $\mathrm{CE}$, Samargandi et al. (2014) estudam o mercado da Arábia Saudita, entre 1968 e 2010 e recorrem ao método estimador ARDL. Concluem que o desenvolvimento do setor financeiro tem um impacto positivo e estatisticamente significante no setor não petrolífero da amostra, enquanto no setor petrolífero os resultados são negativos ou não estatisticamente significantes.

Alguns dos estudos mencionados anteriormente, apenas estudam o tema desenvolvimento-crescimento de maneira linear, como se o processo fosse constante. A partir da década de 90 surgiram estudos com novas técnicas econométricas, originando questões acerca da linearidade do DF sobre CE, sendo que alguns artigos já incluíam determinantes energéticos e ambientais. Deidda e Fattouh (2002) consideraram um painel de 119 países (desenvolvidos e em desenvolvimento) e aplicaram regressões 
threshold (limiar) para a análise da amostra e concluíram que havia uma relação significante entre o DF e o CE nos países de alto rendimento e uma relação estatisticamente insignificante nos países de baixo rendimento. Easterly et al. (2000) concluíram que havia uma relação não linear entre o financiamento e a volatilidade do CE. Observou-se que o crédito bancário cedido ao setor privado gera um obstáculo ao CE se esse crédito ultrapassar 90\% do PIB de uma economia (Cecchiti e Kharroubi, 2012).

O impacto do DF sobre o CE tem manifestado alguma moderação ao longo do tempo. Com base na comparação dos dados em painel de 1990-2004 com 1960-1989, Rousseau e Wachtel (2011) argumentam que o rápido crescimento do crédito e a generalização da liberalização nos anos 90, originado por pressões inflacionárias e enfraquecimento do sistema bancário, leve a que o DF diminua o seu impacto no CE ao longo do tempo. Posteriormente, Kar et al., (2011) argumentam que o nexus desenvolvimento-crescimento em economias desenvolvidas não estabelece nenhuma relação significante. Analisando 52 países com rendimentos médios/baixos, entre 1980 e 2008, Samargandi et al. (2015) mostram a existência de um U invertido, no longo prazo, quando analisam a relação entre Desenvolvimento Financeiro e Crescimento Econômico. A curto prazo a relação é insignificante. Este estudo vai de encontro com o artigo de Arcand et al., (2012).

O sistema financeiro torna-se assim uma questão pertinente na literatura, já que diferentes autores alegam que a partir de um certo limite o desenvolvimento do sistema financeiro deixa de ter um choque positivo e torna-se negativo ou inexistente (Arcand et al., 2012; Cecchetti e Kharroubi, 2012; Rousseau e Wachtel, 2011).

A síntese dos vários artigos, estudos e investigações mais relevantes produzidas ao longo do tempo sobre os temas energia, CE, produção de petróleo e DF, foram descritas na secção presente. Iremos demonstrar na secção seguinte a metodologia e dados do presente artigo.

\section{DADOS E METODOLOGIA}

O objetivo da realização deste trabalho é analisar a relação entre DF e CE em países produtores de petróleo. Embora existam diversos produtores deste tipo de energia fóssil, a obtenção de dados para o horizonte temporal e para as variáveis em questão, é limitada. Utilizando critérios de seriação tendo em perspectiva as necessidades que o estudo exige e empregando séries que contenham toda a informação disponível (sem 
quebras) para o período temporal em análise, os países usados são: Arábia Saudita, Argélia, Austrália, Brasil, Dinamarca, Egito, Equador, Estados Unidos da América, Índia, Indonésia, Itália, Malásia, México, Peru e Tailândia (15). Três dos países que mais petróleo produziram a nível global nos últimos 10 anos $^{3}$ (Canadá, República Popular da China e Rússia), não incorporam o conjunto de países da amostra, devido ao fato de não apresentarem dados suficientes para a construção das variáveis. Os dados inseridos no estudo são anuais e têm um período temporal que se inicia em 1981 e termina em 2011.

Os dados para a construção das variáveis foram retirados das seguintes fontes: The World Data Bank (para PIB, população total, rendas de petróleo e exportações de bens e serviços), BP Statistical Review of World Energy, Junho de 2014 (para consumo, produção e preços de petróleo, consumo de energia primária e emissões de $\mathrm{CO}_{2}$ ) e Fundo Monetário Internacional (para liquid liabilities - M3). A descrição pormenorizada das variáveis utilizadas é a seguinte: (i) PIB (preços constantes da moeda local); (ii) população (número total de pessoas); (iii) rendas de petróleo (\% do PIB); (iv) exportações de bens e serviços (\% do PIB); (v) consumo de petróleo (milhões de toneladas); (vi) produção de petróleo (milhões de toneladas); (vii) consumo de energia primária (milhões de toneladas equivalentes de petróleo); (viii) emissões de $\mathrm{CO}_{2}$ (milhões de toneladas); (ix) preços do petróleo (US dólares de 2013 por barril) e (x) liquid liabilities - M3 (\% do PIB). Os softwares utilizados na análise econométrica foi o Stata 13.1 e EViews 9.

Procedeu-se assim à transformação das seguintes variáveis:

- Produto Interno Bruto per capita (YPC) - obtido através do quociente entre PIB e população total. A utilização de PIB a preços constantes da moeda local serviu para contornar os problemas relacionados com as taxas de câmbio;

- Consumo de petróleo per capita (OCPC) - obtido através do quociente entre consumo de petróleo e população total;

- Rácio entre produção de petróleo e consumo de energia primária (SE) - obtido através do quociente entre produção de petróleo e consumo de energia primária. Segundo Fuinhas et al. (2015) este rácio é utilizado para controlar a heterogeneidade da produção nos produtores de petróleo. Mede também a importância da produção de petróleo em relação à energia primária, expressa em unidades físicas. Além disso, o rácio $\mathrm{SE}$ é adequado para estudar com variáveis per capita e capturar registros de que o consumo de energia não evolui de forma linear em relação à produção de petróleo;

\footnotetext{
${ }^{3}$ Canadá: 3.99\%; República Popular da China: 4.80\%; Rússia: 12.43\% - Percentagem média de produção de petróleo a nível mundial (2003-2013) - Elaboração própria. Dados BP Statistical Review of World Energy, Junho 2014.
} 
- Rendas de petróleo per capita (ORPC) - calculada em três passos. Efetua-se o quociente das rendas de petróleo (\% do PIB) por 100. Em seguida multiplica-se pelo PIB, de forma a obter os valores reais na moeda local. Por último, divide-se pela população total, para a variável ficar per capita. Esta variável é a diferença entre o valor de produção do petróleo bruto a preços mundiais e os seus custos de produção;

- Preço do petróleo $(\mathrm{P})$ - refere-se ao preço internacional de petróleo, medidos em US dólares de 2013 por barril. Esta variável é igual para todos os países. É de esperar que apenas tenha efeitos no curto prazo;

- Exportações de bens e serviços per capita (EXPC) - calculada em três passos. Quociente entre as exportações de bens e serviços (\% do PIB) por 100. Em seguida multiplica-se pelo PIB, para obter os valores reais na moeda local. Por último, divide-se pela população total, para a variável ficar per capita. Esta variável representa os bens e serviços entregues pelos países. É de esperar que esta variável tenha efeitos no curto e longo prazo;

- Emissões de $\mathrm{CO}_{2}$ per capita (CO2PC) - obtido através do quociente entre as emissões de $\mathrm{CO}_{2}$ e população total. Esta variável está relacionada com o consumo de petróleo e o PIB, podendo apresentar problemas de colinearidade;

- Desenvolvimento Financeiro (DF) - calculado em três passos. Multiplicar inicialmente a variável M3 pelo PIB e em seguida dividir por 100, de modo a obter os valores reais. Para tornar a variável num rácio, procede-se assim à sua divisão pelo PIB.

Existem diversas formas para medir o DF através de proxies financeiras. Por exemplo Lean e Smith (2009) utilizam no seu estudo o crédito privado em percentagem do PIB; Sadorsky $(2010$; 2011) recorre ao total dos depósitos bancários em percentagem do PIB, agregado monetário (M3 em \% PIB) e os deposit money bank assests. A Análise de Componentes Principais, é um procedimento matemático que ajuda a medir o DF. Este método agrupa várias variáveis financeiras de forma a contornar o problema da multicolinearidade, extraindo a informação significante do conjunto de dados. Este método é utilizado por alguns autores (e.g. Ang e Mckibbin, 2007; Çoban e Topcu, 2013; Samargandi et al., 2014). No estudo presente emprega-se o M3 por ser uma das proxies que melhor se adequa à mensuração do setor financeiro. Por sugestão de outros autores, procedemos ao rácio $\mathrm{M} 3 / \mathrm{PIB}$, por ser um agregado monetário menos líquido (Beck et al., 2000; Deidda e Fattouh, 2002; Favara, 2003). Este rácio capta a quantidade de passivos líquidos do setor financeiro, como os passivos dos bancos, bancos centrais e outros intermediários financeiros, refletindo assim o DF (King e Levine, 1993a; King e Levine, 1993b; Demetriades e Hussein, 1996).

Como o período temporal é longo, espera-se que as variáveis em estudo apresentem efeitos dinâmicos, tal como expetáveis comportamentos diferentes, quer no curto prazo como no longo prazo. Em consequência destas proposições, será necessário 
proceder a técnicas econométricas adequadas, utilizando assim o estimador ARDL. Este estimador é conhecido pela sua eficiência e consistência na estimação de variáveis enquanto $\mathrm{I}(0)$ e $\mathrm{I}(1)$ e tem como vantagem suportar a inferência de parâmetros com base em testes padrão.

Todas as variáveis encontram-se em logaritmos naturais (com o prefixo "L"), à exceção do Desenvolvimento Financeiro (DF), e primeiras diferenças (com o prefixo “D”). As variáveis em nível e diferenças são de longo e curto prazo, respetivamente. Deste modo, os primeiros coeficientes correspondem às elasticidades e os segundos coeficientes às semi-elasticidades. Segue a especificação do modelo ARDL, Eq.(1):

$$
\begin{aligned}
& L_{Y P C} C_{i t}=\alpha_{i}+\beta_{i 1} L Y P C_{i t-1}+\beta_{i 2} S E_{i t}+\beta_{i 3} S E_{i t-1}+\beta_{i 4} L O R P C_{i t}+\beta_{i 5} L O R P C_{i t-1}+ \\
& +\beta_{i 6} L P_{i t}+\beta_{i 7} L P_{i t-1}+\beta_{i 8} D F_{i t}+\beta_{i 9} D F_{i t-1}+\beta_{i 10} L E X P C_{i t}+\beta_{11} L E X P C_{i t-1}+ \\
& +\beta_{i 12} L C O 2 P C_{i t}+\beta_{i 13} L C O 2 P C_{i t-1}+\beta_{i 14} L O C P C_{i t}+\beta_{i 15} L O C P C_{i t-1}+\varepsilon_{i t}
\end{aligned}
$$

A Eq.(1) pode ser reformulada de modo a decompor a relação dinâmica existente entre as variáveis, seja a longo e curto prazo, do seguinte modo, Eq.(2):

$$
\begin{aligned}
& D L Y P_{i t}=\alpha_{2 i}+\beta_{i 1} D S E_{i t}+\beta_{i 2} D_{L O R P C}+\beta_{i 3} D L P_{i t}+\beta_{i 4} D D F_{i j}+\beta_{i 5} D E X P C_{i t}+ \\
& +\beta_{i 6} L C O 2 P C_{i t}+\beta_{i 7} L O C P C_{i t-1}+\gamma_{i 1} L Y P C_{i t-1}+\gamma_{i 2} S_{i t-1}+\gamma_{i 3} L O R P C_{i t-1}+ \\
& +\gamma_{i 4} D F_{i t-1}+\gamma_{i 5} L E X P C_{i t-1}+\gamma_{i 6} L C O 2 P C_{i t-1}+\gamma_{i 7} L O C P C_{i t-1}+\varepsilon_{i t}
\end{aligned}
$$

onde $\alpha$ corresponde à constante; $\beta_{i}$ e $\gamma_{i}$ aos parâmetros estimados; $\varepsilon_{i}$ ao termo de erro.

\subsection{Análise preliminar dos Dados}

A análise preliminar dos dados em estudo é necessária para uma melhor compreensão das caraterísticas de todas as séries. O estudo em causa é realizado com um macro painel (15 países) e com um horizonte temporal entre 1981 e 2011, tais fatos levam-nos a questionar a existência de choques comuns entre as variáveis em análise. $\mathrm{O}$ tema do artigo reflete um conjunto de países, que possuem linhas orientadoras comuns, como a produção de petróleo. Deste modo, é de esperar Cross Section Dependence (CSD), que segundo Eberhardt (2011) este fenômeno implica uma interdependência entre as crosses devido a choques comuns. CSD ocorre normalmente em macro painéis, e pode levar à existência de ineficiências e distorções nas estimativas, se este não for tratado adequadamente.

É importante analisar as estatísticas descritivas das variáveis, o CSD e a ordem de integração das mesmas, para capturar as caraterísticas das séries e o grau de dependência entre as variáveis. Portanto, a Tabela 1 apresenta as estatísticas descritivas e o teste CSD das variáveis. 
Tabela 1. Descrição das variáveis e CSD

\begin{tabular}{|c|c|c|c|c|c|c|c|c|}
\hline \multirow[b]{2}{*}{ Variável } & \multicolumn{5}{|c|}{ Descrição das variáveis } & \multicolumn{3}{|c|}{$\begin{array}{l}\text { Cross section dependence } \\
\text { (CSD) }\end{array}$} \\
\hline & $\begin{array}{c}\mathrm{Ob} \\
\mathrm{s}\end{array}$ & Média & Dsv Pad & Min & Max & CD-test & corr & abs (corr) \\
\hline LYPC & 465 & 10.1247 & 1.9401 & 6.8194 & 16.1289 & $\begin{array}{c}40.05^{* *} \\
*\end{array}$ & $\begin{array}{c}0.702 \\
0\end{array}$ & 0.7150 \\
\hline $\mathrm{SE}$ & 465 & 1.0292 & 1.1978 & 0.0054 & 10.9920 & $4.15^{* * *}$ & $\begin{array}{c}0.073 \\
0\end{array}$ & 0.6500 \\
\hline LORPC & 465 & 6.3727 & 2.6309 & -2.4714 & 13.4694 & $\begin{array}{c}34.10^{* *} \\
*\end{array}$ & $\begin{array}{c}0.598 \\
0\end{array}$ & 0.6240 \\
\hline LP & 465 & 3.7985 & 0.4927 & 2.8994 & 4.7469 & $\begin{array}{c}57.05^{* *} \\
*\end{array}$ & $\begin{array}{c}1.000 \\
0\end{array}$ & 1.0000 \\
\hline DF & 465 & 0.5489 & 0.2780 & 0.0650 & 1.3234 & $\begin{array}{c}18.58 * * \\
*\end{array}$ & $\begin{array}{c}0.326 \\
0\end{array}$ & 0.4380 \\
\hline LEXPC & 465 & 8.6835 & 2.0969 & 5.2845 & 15.0479 & $\begin{array}{c}41.82^{* *} \\
*\end{array}$ & $\begin{array}{c}0.733 \\
0\end{array}$ & 0.7330 \\
\hline $\mathrm{LCO} 2 \mathrm{PC}$ & 465 & -12.5413 & 1.0932 & -14.5131 & -10.6975 & $\begin{array}{c}21.56^{* *} \\
*\end{array}$ & $\begin{array}{c}0.378 \\
0\end{array}$ & 0.6110 \\
\hline LOCPC & 465 & -14.1958 & 1.0355 & -16.8605 & -12.2986 & $6.31 * * *$ & $\begin{array}{c}0.111 \\
0\end{array}$ & 0.4720 \\
\hline DLYPC & 450 & 0.0186 & 0.0374 & -1.1812 & 0.1071 & $5.65 * * *$ & $\begin{array}{c}0.101 \\
0\end{array}$ & 0.1880 \\
\hline DSE & 450 & -0.0349 & 0.2777 & -4.2632 & 1.3663 & 0.23 & $\begin{array}{c}0.004 \\
0\end{array}$ & 0.2330 \\
\hline DLORPC & 450 & 0.0195 & 0.3471 & -1.1778 & 2.8713 & $\begin{array}{c}41.01 * * \\
*\end{array}$ & $\begin{array}{c}0.731 \\
0\end{array}$ & 0.7310 \\
\hline DLP & 450 & 0.0075 & 0.2533 & -0.6655 & 0.4280 & $\begin{array}{c}56.12 * * \\
*\end{array}$ & $\begin{array}{c}1.000 \\
0\end{array}$ & 1.0000 \\
\hline DDF & 450 & 0.0101 & 0.0475 & -0.2798 & 0.2206 & $6.06 * * *$ & $\begin{array}{c}0.108 \\
0\end{array}$ & 0.2230 \\
\hline DLEXPC & 450 & 0.0333 & 0.1327 & -0.6327 & 0.5591 & $\begin{array}{c}14.71 * * \\
*\end{array}$ & $\begin{array}{c}0.262 \\
0\end{array}$ & 0.3120 \\
\hline $\begin{array}{l}\text { DLCO2P } \\
\mathrm{C}\end{array}$ & 450 & 0.0179 & 0.0500 & -0.1821 & 0.1836 & $2.34 * *$ & $\begin{array}{c}0.042 \\
0\end{array}$ & 0.1920 \\
\hline DLOCPC & 450 & 0.0078 & 0.0478 & -0.1874 & 0.1839 & $2.03 * *$ & $\begin{array}{c}0.036 \\
0\end{array}$ & 0.2020 \\
\hline
\end{tabular}

Notas: O prefixo "L" significa logaritmo natural e o " $\mathrm{D}$ " significa primeiras diferenças. $\mathrm{O}$ teste CSD tem distribuição $\mathrm{N}(0,1)$ e a $\mathrm{H}_{0}$ : cross-section dependence. *** e ** denotam significância de $1 \%$ e $5 \%$, respetivamente. O comando utilizado no software Stata para testar a CSD foi $x t c d$.

Note-se na Tabela 1, a clara evidência de diversificação no macro painel. Verifica-se também grande dependência entre as variáveis, apresentando níveis de significância entre $1 \%$ e 5\%, provando que qualquer choque que afete um país deste estudo, também irá afetar os restantes, seja negativamente ou positivamente. Existe 
apenas uma variável (DSE) que não apresenta dependência secional. Essa ausência sugere que os países deste estudo reagem de forma independente em relação à produção de petróleo e consumo de energia (Fuinhas et al, 2015).

Um outro problema, enunciado na literatura econométrica, é a presença de colinearidade, isto é, a correlação entre diferentes variáveis, pois mesmo sendo de naturezas diferentes podem explicar da mesma maneira a variável dependente, apresentando características de variáveis substitutas uma da outra. O problema reside no fato de a variância dos coeficientes da regressão em causa serem inflacionados, originando erros na significância dos preditores (Dormann et al., 2013). A Tabela 2 apresenta a matriz de correlações das variáveis em análise e os seus respetivos fatores de inflação da variância (VIF - Variance Inflator Factor).

Tabela 2. Matriz das correlações e estatísticas VIF

\begin{tabular}{|c|c|c|c|c|c|c|c|c|}
\hline & LYPC & $\mathrm{SE}$ & LORPC & $\overline{\mathrm{LP}}$ & $\overline{\mathrm{DF}}$ & LEXPC & LCO2PC & $\mathrm{LOCPC}$ \\
\hline LYPC & 1.0000 & & & & & & & \\
\hline SE & $\begin{array}{c}- \\
0 .\end{array}$ & $\begin{array}{c}1.000 \\
0\end{array}$ & & & & & & \\
\hline LORPC & 0.7554 & $\begin{array}{c}0.456 \\
4\end{array}$ & 1.0000 & & & & & \\
\hline LP & 0.0278 & $\begin{array}{c}- \\
0.019 \\
5\end{array}$ & 0.1528 & $\begin{array}{c}1.000 \\
0\end{array}$ & & & & \\
\hline DF & 0.1585 & $\begin{array}{c}- \\
0.289 \\
6\end{array}$ & -0.2096 & $\begin{array}{c}0.074 \\
0\end{array}$ & 1.0000 & & & \\
\hline LEXPC & 0.9521 & $\begin{array}{c}0.044 \\
8\end{array}$ & 0.7795 & $\begin{array}{c}0.064 \\
5\end{array}$ & $\begin{array}{c}- \\
0.0024\end{array}$ & 1.0000 & & \\
\hline $\mathrm{LCO} 2 \mathrm{PC}$ & 0.1698 & $\begin{array}{c}0.026 \\
3\end{array}$ & 0.0170 & $\begin{array}{c}0.025 \\
3\end{array}$ & 0.3350 & 0.2126 & 1.0000 & \\
\hline LOCPC & 0.0784 & $\begin{array}{c}0.123 \\
4\end{array}$ & -0.0251 & $\begin{array}{c}0.009 \\
6\end{array}$ & 0.2425 & 0.1426 & 0.9453 & 1.0000 \\
\hline VIF & & $\begin{array}{l}2.46 \\
2.10 \\
2.28 \\
\end{array}$ & $\begin{array}{l}6.32 \\
5.74 \\
6.09 \\
\end{array}$ & $\begin{array}{l}1.09 \\
1.09 \\
1.09 \\
\end{array}$ & $\begin{array}{l}1.34 \\
1.28 \\
1.22 \\
\end{array}$ & $\begin{array}{l}4.72 \\
4.59 \\
4.72 \\
\end{array}$ & $\begin{array}{c}12.35 \\
1.33\end{array}$ & $\begin{array}{l}12.31 \\
1.33 \\
\end{array}$ \\
\hline $\begin{array}{l}\text { VIF - } \\
\text { Média }\end{array}$ & \multicolumn{8}{|c|}{ Global $=5.8 ;$ Modelo $1=2.69 ;$ Modelo $2=2.79$} \\
\hline & $\begin{array}{l}\text { DLYP } \\
\text { C }\end{array}$ & DSE & $\begin{array}{l}\text { DLORP } \\
\mathrm{C}\end{array}$ & DLP & DDF & $\begin{array}{l}\text { DLEXP } \\
\text { C }\end{array}$ & $\begin{array}{l}\mathrm{DLCO} 2 \mathrm{P} \\
\mathrm{C}\end{array}$ & $\begin{array}{l}\text { DLOCP } \\
\text { C }\end{array}$ \\
\hline DLYPC & 1.0000 & & & & & & & \\
\hline DSE & 0.3462 & $\begin{array}{c}1.000 \\
0\end{array}$ & & & & & & \\
\hline DLORPC & 0.1773 & 0.159 & 1.0000 & & & & & \\
\hline
\end{tabular}




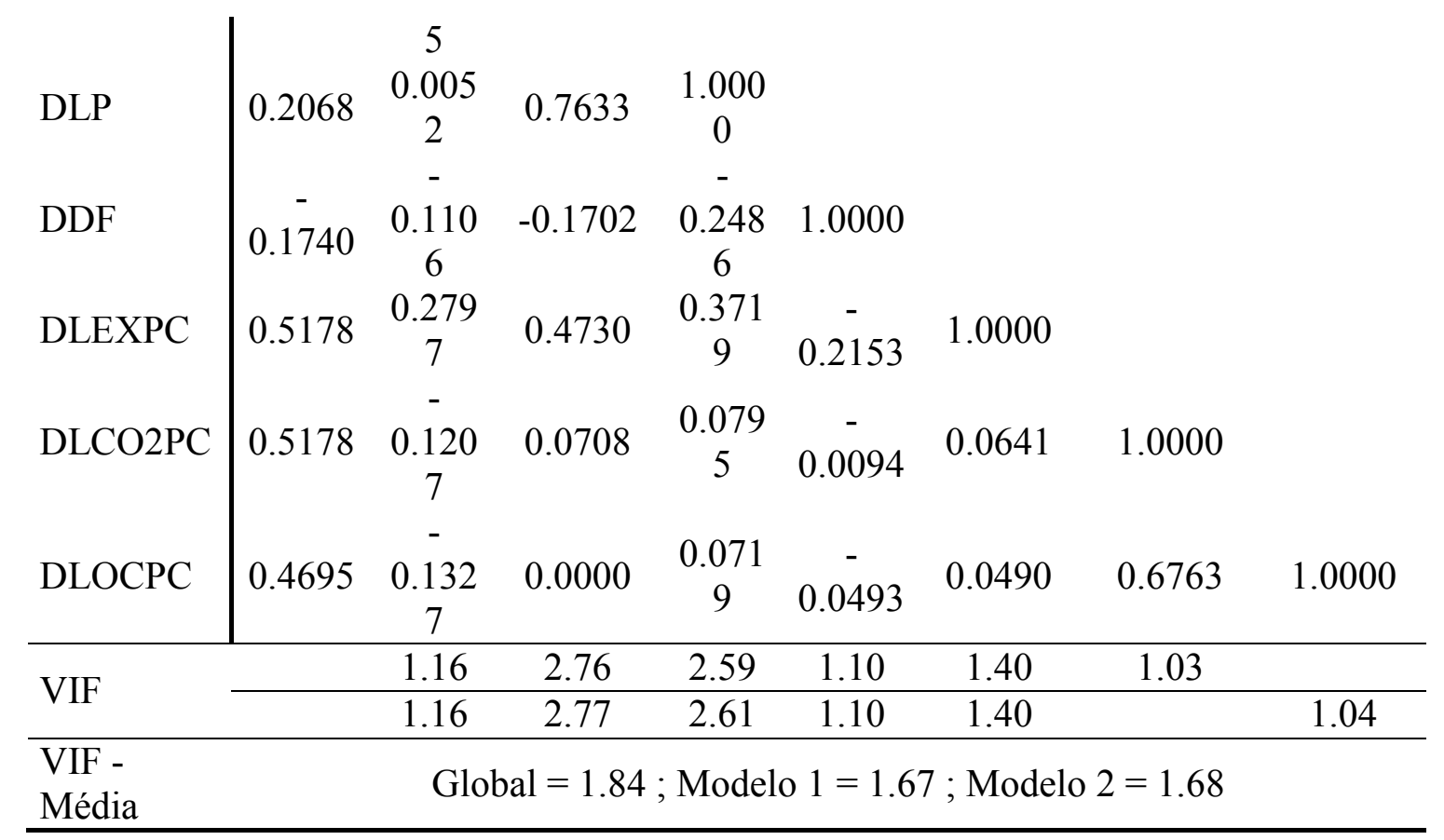

$\mathrm{Na}$ Tabela 2 pode-se notar que apenas duas variáveis apresentam valores não desejados, isto porque os seus coeficientes de correlação linear são maiores do que 0.8 , nomeadamente entre as variáveis LEXPC/LYPC e LOCPC/LCO2PC. Ao observarmos os valores VIF, pode-se afirmar que o problema entre LEXPC e LYPC é resolvido, pois o valor da variável independente (LEXPC) e dependente (LYPC) é menor que 10, sendo este um requisito para a não existência de multicolinearidade. Com as outras variáveis (LOCPC e $\mathrm{LCO}_{2} \mathrm{PC}$ ) o fenômeno de multicolinearidade é evidente, pois os valores VIF são maiores que 10. Este problema entre estas duas variáveis colineares reside no fato de elas explicarem da maneira similar o PIB, apresentam caraterísticas de variáveis substitutas uma da outra.

Assim, para contornar este problema separaram-se as variáveis e formaram-se dois modelos: o Modelo 1, com as emissões de $\mathrm{CO}_{2}$ per capita (LCO2PC), Eq.(3):

$$
\begin{aligned}
& D L Y P_{i t}=\alpha_{3 i}+\beta_{i 1} D S E_{i t}+\beta_{i 2} D L O R P C_{i t}+\beta_{i 3} D L P_{i t}+\beta_{i 4} D D F_{i j}+\beta_{i 5} D L E X P C_{i t}+ \\
& +\beta_{i 6} D C O 2 P C_{i t}+\gamma_{i 1} L Y P C_{i t-1}+\gamma_{i 2} S E_{i t-1}+\gamma_{i 3} L O R P C_{i t-1}+\gamma_{i 4} D F_{i t-1}+ \\
& +\gamma_{i 5} L E X P C_{i t-1}+\gamma_{i 6} L C O 2 P C_{i t-1}+\varepsilon_{i t}
\end{aligned}
$$

E o Modelo 2, com o consumo de petróleo per capita (LOCPC), Eq(4): 


$$
\begin{aligned}
& D L Y P_{i t}=\alpha_{4 i}+\beta_{i 1} D S E_{i t}+\beta_{i 2} D L O R P C_{i t}+\beta_{i 3} D L P_{i t}+\beta_{i 4} D D F_{i j}+\beta_{i 5} D E X P C_{i t}+ \\
& +\gamma_{i 6} D O C P C+\gamma_{i 1} L Y P C_{i t-1}+\gamma_{i 2} S E_{i t-1}+\gamma_{i 3} L O R P C_{i t-1}+\gamma_{i 4} D F_{i t-1}+ \\
& +\gamma_{i 5} L E X P C_{i t-1}+\gamma_{i 6} L O C P C+\varepsilon_{i t}
\end{aligned}
$$

onde $\alpha$ corresponde à constante; $\beta_{i}$ e $\gamma_{i}$ aos parâmetros estimados; $\varepsilon_{i}$ ao termo de erro.

Com estes dois modelos o problema de multicolinearidade entre as variáveis deixa de existir. Para podermos testar a presença de raízes unitárias nas variáveis em estudo, procedeu-se aos testes de primeira e segunda geração. LLC (Levin et al., 2002), Breitung (2000), IPS (Im et al., 2003), ADF-Fisher (Maddala e Wu, 1999) e ADF-Choi (Choi, 2001) foram os testes de primeira geração de raízes unitárias realizados. Os

\begin{tabular}{|c|c|c|c|c|c|c|c|c|c|}
\hline \multirow[b]{3}{*}{ Variável } & \multicolumn{9}{|c|}{$1^{\mathrm{a}}$ geração } \\
\hline & \multicolumn{2}{|c|}{ LLC } & \multirow{2}{*}{$\begin{array}{c}\text { Breitung } \\
\text { CT }\end{array}$} & \multicolumn{2}{|c|}{ IPS } & \multicolumn{2}{|c|}{ ADF-Fisher } & \multicolumn{2}{|c|}{ ADF-Choi } \\
\hline & $\mathrm{CT}$ & $\mathrm{CC}$ & & $\mathrm{CT}$ & $\mathrm{CC}$ & $\mathrm{CT}$ & $\mathrm{CC}$ & CT & $\mathrm{CC}$ \\
\hline LYPC & 0.494 & -0.151 & 3.222 & 1.533 & 3.740 & 22.593 & 17.916 & 1.604 & 3.590 \\
\hline $\mathrm{SE}$ & 1.495 & $-3.237 * * *$ & -0.041 & -1.031 & -0.488 & $45.849 * *$ & 38.946 & -1.059 & -0.436 \\
\hline LORPC & $-3.403 * * *$ & $-1.799 * *$ & -0.119 & -1.124 & -1.042 & $43.139 *$ & 34.664 & -0.855 & -1.030 \\
\hline LP & $-4.457 * * *$ & 2.812 & 5.071 & 3.183 & 2.439 & 6.014 & 8.234 & 3.521 & 2.735 \\
\hline DF & 0.060 & 0.323 & -0.354 & -0.414 & -0.002 & 36.917 & 33.271 & -0.394 & -0.055 \\
\hline LEXPC & $-1.343^{*}$ & -1.001 & -0.167 & $-1.2861^{*}$ & 2.417 & $46.357 * *$ & 13.150 & $-1.296^{*}$ & 2.499 \\
\hline $\mathrm{LCO} 2 \mathrm{PC}$ & 2.345 & $-1.391^{*}$ & 2.372 & 2.516 & 3.133 & 16.366 & 13.224 & 2.634 & 3.160 \\
\hline LOCPC & 2.546 & -0.206 & 0.122 & 1.605 & 1.684 & 25.206 & 25.455 & 1.656 & 1.736 \\
\hline DLYPC & $-7.957 * * *$ & $-8.065 * * *$ & 4. $\overline{11}$ * $* * *$ & $-7.039 * * *$ & $-7.618 * * *$ & $102.300 * * *$ & $115.733 * * *$ & $-6.837 * * *$ & $-7.522 * * *$ \\
\hline DSE & $-6.572 * * *$ & $-8.228 * * *$ & $\begin{array}{c}- \\
4.313 * * *\end{array}$ & $\begin{array}{c}- \\
11.147 * * *\end{array}$ & $\begin{array}{c}- \\
11.654 * * *\end{array}$ & $167.059 * * *$ & $183.655^{* * *}$ & $-9.668 * * *$ & $10.536^{* * *}$ \\
\hline DLORPC & $-9.318 * * *$ & $\begin{array}{c}- \\
11.849 * * *\end{array}$ & $\begin{array}{c}- \\
7.946^{* * *}\end{array}$ & $\begin{array}{c}- \\
11.938^{*} * *\end{array}$ & $12.849^{* * *}$ & $174.577 * * *$ & $203.154 * * *$ & $\begin{array}{c}- \\
10.549 * * *\end{array}$ & $\begin{array}{c}- \\
11.722 * * *\end{array}$ \\
\hline DLP & $\begin{array}{c}- \\
12.610^{* * *}\end{array}$ & $10.431^{-} * * *$ & $5.130^{-} * * *$ & $\begin{array}{c}- \\
13.639 * * *\end{array}$ & $\begin{array}{c}- \\
10.152^{* * *}\end{array}$ & $201.150 * * *$ & $155.062 * * *$ & $\stackrel{-}{11.733 * * *}$ & $-9.801 * * *$ \\
\hline DDF & $-5.192 * * *$ & $-6.041 * * *$ & $\begin{array}{c}- \\
3.746 * * *\end{array}$ & $-5.903 * * *$ & $-7.212 * * *$ & $89.143 * * *$ & $112.468 * * *$ & $-5.728 * * *$ & $-7.049 * * *$ \\
\hline DLEXPC & $-7.725 * * *$ & $-9.869 * * *$ & $\begin{array}{c}- \\
5.149 * * *\end{array}$ & $-9.599 * * *$ & $10.967 * * *$ & $139.620 * * *$ & $171.254 * * *$ & $-8.819 * * *$ & $10.236^{* * *}$ \\
\hline DLCO2PC & $-7.418 * * *$ & $-7.395 * * *$ & $\begin{array}{c}- \\
3.963 * * *\end{array}$ & $-9.948 * * *$ & $10.198 * * *$ & $146.243 * * *$ & $158.906^{* * *}$ & $-8.927 * * *$ & $-9.581 * * *$ \\
\hline DLOCPC & $-4.646^{* * *}$ & $-5.578 * * *$ & $\begin{array}{c}- \\
3.417 * * * \\
\end{array}$ & $-7.650 * * *$ & $-8.426 * * *$ & $112.440 * * *$ & 129.634 & $-7.201 * * *$ & $-8.149 * * *$ \\
\hline
\end{tabular}
resultados dos testes de raízes unitárias são apresentados na Tabela 3.

Tabela 3. Testes de raízes unitárias $-\mathbf{1}^{\mathrm{a}}$ geração

Notas: $* * * * * \mathrm{e}^{*}$ denotam significância de $1 \%, 5 \%$ e $10 \%$, respetivamente. $\mathrm{CT}$ ) representa o teste unit root com constante e tendência. CC) representa o teste unit root apenas com constante. As hipóteses nulas $\left(\mathrm{H}_{0}\right)$ são: LLC: painéis 
contêm raiz unitária; Breitung: painéis contêm raiz unitária; IPS: todos os painéis contêm raiz unitária; ADF-Fisher e ADF-Choi: painéis contêm raiz unitária. Os testes foram realizados no software EViews 9.

Verifica-se que as variáveis em diferenças e em nível são $\mathrm{I}(0)$ e $\mathrm{I}(1)$, respetivamente, nos testes IPS, Breitung, ADF-Fisher e ADF-Choi. Realizaram-se também os testes de raízes unitárias de segunda geração (CIPS) segundo Pesaran (2007), (ver Tabela 4). Este teste tem como vantagem de ser robusto para a heterogeneidade e testa a hipótese nula $\left(\mathrm{H}_{0}\right)$ de não estacionariedade.

Tabela 4. Testes de raízes unitárias (CIPS) $-2^{\mathrm{a}}$ geração

\begin{tabular}{|c|c|c|}
\hline \multirow[b]{3}{*}{ Variável } & \multicolumn{2}{|c|}{$2^{\mathrm{a}}$ geração } \\
\hline & \multicolumn{2}{|c|}{ CIPS (Zt-bar) } \\
\hline & Sem tendência & $\begin{array}{c}\text { Com } \\
\text { tendência }\end{array}$ \\
\hline LYPC & 0.092 & 1.273 \\
\hline SE & 0.018 & 0.067 \\
\hline LORPC & $-3.255^{* * *}$ & $-3.352 * * *$ \\
\hline LP & 18.046 & 17.781 \\
\hline DF & -0.024 & 1.17 \\
\hline LEXPC & 0.529 & 0.822 \\
\hline $\mathrm{LCO} 2 \mathrm{PC}$ & 2.295 & 4.111 \\
\hline LOCPC & 0.712 & 1.906 \\
\hline DLYPC & $-4.603 * * *$ & $-3.532 * * *$ \\
\hline DSE & $-8.610 * * *$ & $-8.275^{* * *}$ \\
\hline DLORPC & $-8.513 * * *$ & $-6.795 * * *$ \\
\hline DLP & 17.488 & 16.741 \\
\hline DDF & $-4.376 * * *$ & $-3.806^{* * *}$ \\
\hline DLEXPC & $-6.542 * * *$ & $-5.694 * * *$ \\
\hline DLCO2PC & $-7.225 * * *$ & $-7.111 * * *$ \\
\hline DLOCPC & $-5.785 * * *$ & $-5.271 * * *$ \\
\hline $\begin{array}{l}\text { Notas: ***, } \\
\text { respetivame } \\
\text { unitária em } \\
\text { foi incluído } \\
\text { aos cálculos. }\end{array}$ & $\begin{array}{l}\text { significância } \\
\text { a }\left(\mathrm{H}_{0}\right) \text { é: Pesa } \\
\text { séries são I(1) } \\
\text { tlizado o softw } \\
\text { ndo multipurt. }\end{array}$ & $\begin{array}{l}5 \% \text { e } 10 \%, \\
\text { teste de raiz } \\
\text { ste realizado } \\
\text { ara proceder }\end{array}$ \\
\hline
\end{tabular}

Pode-se observar que o teste de segunda geração de raízes unitárias indica para que a variável LORPC seja I(0). A variável preços de petróleo (LP) é ser comum a todas as crosses. As restantes levam à aceitação da hipótese nula, sendo assim as variáveis são I(1). Ou seja, o problema de existir integração de segunda ordem, I(2), não se coloca em questão. Devido à ambiguidade dos resultados encontrados no teste CIPS e nos testes de primeira geração (LLC, ADF-Fisher e ADF-Choi) e à existência de CSD nas variáveis, optou-se por realizar o teste de cointegração Westerlund (2007). Este último teste 
permite verificar a existência de cointegração no painel e por crosses e é robusto na presença de CSD devido a permitir a utilização da técnica de bootstrapping para corrigir os níveis de significância dos parâmetros estimados. Os resultados do teste Westerlund demonstram a não cointegração das variáveis. Este resultado pode decorrer do fato de as variáveis não serem integradas de ordem I(1). Como se pode observar nas Tabelas 3 e 4, os testes de raízes unitárias não são conclusivos relativamente à ordem de cointegração de algumas variáveis.

A existência de um extenso período de tempo e um macro painel de países produtores de petróleo, segundo as boas práticas econométricas, requer a utilização de técnicas que possam lidar com o fenômeno da heterogeneidade e a decomposição de fenômenos de curto e longo prazo. Assim o passo seguinte é verificar qual o melhor estimador dos modelos através do teste Hausman, testando a presença de efeitos fixos (FE) contra efeitos aleatórios (RE). Partimos do pressuposto que nos FE a heterogeneidade dos países consegue-se observar no termo constante, sendo este diferente de país para país. No estimador RE o coeficiente do termo independente de uma unidade individual é uma extração aleatória de uma população maior (Green, 2008).

De acordo com o teste Hausman, ou seja, FE contra RE, a hipótese nula diz que o melhor modelo a utilizar é o de efeitos aleatórios. Se o teste provar que o RE é o mais apropriado, é necessário fazer mais testes com a regressão OLS para determinar qual o melhor modelo. A estatística do teste Hausman demonstra que o estimador mais adequado é o de FE ( $\chi_{11}^{2}=75.13$, para o Modelo 1; e $\chi_{11}^{2}=63.29$, para o Modelo 2), levando à rejeição da hipótese nula. Por outras palavras, há sinais de correlação entre os países de efeitos individuais e as variáveis dependentes.

\section{RESULTADOS}

O fato de se recorrer aos dados em painel para o estudo realizado, deve-se ao elevado número de observações e países. A utilização de macro painéis permite controlar o fenômeno de heterogeneidade. Assim, dados em painel oferecem mais informação, variabilidade, graus de liberdade, eficiência e por consequência menos colinearidade entre as variáveis, sendo que este problema está presente frequentemente nas séries temporais. Possui a capacidade de detectar e medir fenômenos que com outras técnicas econométricas não seria possível efetuar (Klevmarken, 1989; Hsiao-Ping e 
Chang, 2003). Uma outra vantagem deste processo é que proporciona a realização de testes de raiz unitária para ter uma distribuição assintótica normal (Baltagi, 2005).

Após a conclusão que o período temporal é longo e os resultados do teste Hausman provarem a existência de efeitos fixos, é necessário efetuar mais testes de forma a perceber qual o estimador mais adequado para os modelos.

É necessário aplicar dois modelos, Mean Group (MG) e PMG. O modelo MG é o mais flexível, pois permite que os coeficientes sejam heterogéneos entre os vários países. Este modelo é eficaz quando faz estimações no longo-prazo mas é ineficiente quando existe homogeneidade (Pesaran et al., 1999). Um requisito para uma boa estimação com este modelo é a existência de um elevado número de países no painel, 20 a 30 países (Ciarlone, 2011). PMG é também um modelo flexível embora seja menos que o MG. Este modelo executa restrições entre todos os coeficientes de longo prazo, permitindo que no curto prazo exista heterogeneidade nos coeficientes e velocidade de ajustamento, enquanto no longo prazo os coeficientes sejam homogéneos. Se houver presença de homogeneidade, o modelo PMG é o mais consistente em comparação ao MG. A literatura mais recente recorre frequentemente ao método GMM para dados em painel, incluindo áreas financeiras (e.g., Arcand et al, 2012). Com um N pequeno, o método GMM pode criar resultados espúrios e com duplo sentido (Roodman, 2006).

Portanto, o passo seguinte consiste em retirar informações acerca do melhor estimador a utilizar (ver Tabela 5), testando os modelos MG, PME e FE entre eles e ilustrando os respetivos testes Hausman, para prosseguirmos o nosso estudo de forma econometricamente adequada.

Tabela 5. Estimadores heterogéneos e testes Hausman

\begin{tabular}{|c|c|c|c|c|c|c|}
\hline \multicolumn{7}{|c|}{ Variável dependente: DLYPC } \\
\hline \multirow[b]{2}{*}{ Variável } & \multicolumn{3}{|c|}{ Modelo 1} & \multicolumn{3}{|c|}{ Modelo 2} \\
\hline & $\mathrm{MG}$ & PMG & FE & $\mathrm{MG}$ & PMG & $\mathrm{FE}$ \\
\hline Constante & $5.4771 * * *$ & $0.0349 * * *$ & $1.3628 * * *$ & $2.5955^{* *}$ & 0.5759 & $1.0577 * * *$ \\
\hline DSE & $0.2147 *$ & 0.0892 & $0.0609 * * *$ & 0.0354 & -0.0151 & $0.0547 * * *$ \\
\hline DLORPC & ${ }^{-}-0.0635^{* * *}$ & $-0.0456^{* *}$ & $0.0307 * * *$ & $0.0536 * * *$ & -0.0262 & $0.0239 * * *$ \\
\hline DLP & $0.0840 * * *$ & $0.0532 * *$ & $0.0522 * * *$ & $0.0770 * * *$ & $0.0392 *$ & $0.0479 * * *$ \\
\hline DDF & $-0.1106^{*}$ & -0.0953 & $\begin{array}{c}- \\
0.0697 * * *\end{array}$ & $-0.1942 * *$ & $-0.1241 *$ & $\begin{array}{c}- \\
0.0719 * * *\end{array}$ \\
\hline DLEXPC & $0.0536^{*}$ & 0.0388 & $0.0242 * *$ & $0.0627 * *$ & 0.0442 & $0.0206^{*}$ \\
\hline DLCO2PC & $0.3724 * * *$ & $0.4091 * * *$ & $0.3709 * * *$ & & & \\
\hline DLOCPC & & & & $0.2734 * * *$ & $0.3181 * * *$ & $0.3532 * * *$ \\
\hline ECM & - & -0.0078 & - & - & -0.0471 & - \\
\hline
\end{tabular}




\begin{tabular}{|c|c|c|c|c|c|c|}
\hline & $0.3598 * * *$ & & $0.1258 * * *$ & $0.2257 * * *$ & & $0.1038 * * *$ \\
\hline SE & -0.4242 & -0.0004 & $0.0768 * * *$ & -1.5755 & 0.0221 & 0.0202 \\
\hline LORPC & -0.0762 & -0.1796 & $0.1317 * * *$ & 0.0103 & $0.0809 * * *$ & $0.1252 * * *$ \\
\hline LP & 0.0216 & -0.1629 & $0.1239 * * *$ & -0.0688 & 0.0005 & $0.1397 * * *$ \\
\hline DF & 0.2420 & 0.4669 & -0.0770 & 0.5878 & -0.0278 & -0.1566 \\
\hline LEXPC & $0.3018 * * *$ & $1.3482 * *$ & $0.4058 * * *$ & $0.3766^{*}$ & $0.6608 * * *$ & $0.4630 * * *$ \\
\hline $\mathrm{LCO} 2 \mathrm{PC}$ & $0.3675 * * *$ & 0.0430 & $0.3019 * * *$ & & & \\
\hline LOCPC & & & & -0.0886 & $0.5284 * * *$ & $0.2520 * *$ \\
\hline $\begin{array}{l}\text { Teste } \\
\text { Hausman }\end{array}$ & $\begin{array}{l}\text { MG vs } \\
\text { PMG }\end{array}$ & $\begin{array}{c}\text { PMG vs } \\
\text { FE }\end{array}$ & MG vs FE & $\begin{array}{l}\text { MG vs } \\
\text { PMG }\end{array}$ & $\begin{array}{c}\text { PMG vs } \\
\text { FE }\end{array}$ & MG vs FE \\
\hline Chi2(14) & -196.9700 & 0.1000 & 0.0000 & -76.6000 & 0.0000 & 0.0000 \\
\hline Prob $>$ Chi 2 & n.d & 1.0000 & 1.0000 & n.d & 1.0000 & 1.0000 \\
\hline
\end{tabular}

Notas: $* * *, * *$ e $*$ denotam significância de $1 \%, 5 \%$ e $10 \%$, respetivamente. A hipótese nula do teste Hausman $\left(\mathrm{H}_{0}\right)$ : diferença entre os coeficientes não é sistemática. N.d significa não disponível. ECM denota error correction mechanism. O comando utilizado no software Stata foi xtpmg.

Os resultados levam à rejeição dos modelos mais flexíveis (MG e PMG), concluindo que o estimador $\mathrm{FE}$ é o mais adequado, rejeitando a presença de heterogeneidade da inclinação dos parâmetros. O sinal negativo de $\mathrm{X}^{2}$, no teste Hausman, embora seja pouco usual (ver e.g., Dincecco, 2010), realça a rejeição do primeiro estimador testado, tal como indicado por Hausman (1984).

As boas práticas econométricas aconselham a proceder alguns testes no sentido de identificar a existência de violações dos pressupostos no sentido de especificação, para obter informações complementares para se escolher qual o estimador mais adequado. O primeiro teste realizado foi o de Wald. Este teste serve para controlar a heterocedasticidade dos resíduos. Em seguida realizou-se o teste Pesaran e Friedman, para analisar a dependência transversal dos erros. Efetuou-se também o teste BreuschPagan Lagrangian Multiplier, com o intuito de testar a independência entre as crosses e verificar se existe correlação entre os erros. Finalmente, procedeu-se ao teste Wooldridge, de modo a aferir a existência de correlação serial. Os resultados dos testes estão evidenciados na Tabela 6.

Tabela 6. Testes de especificação

\begin{tabular}{lcc}
\hline \multirow{2}{*}{ Teste } & Modelo 1 & Modelo 2 \\
\cline { 2 - 3 } & \multicolumn{2}{c}{ Estatísticas } \\
\hline Teste de Wald Modificado & $336.81^{* * *}$ & $253.17^{* * *}$ \\
Teste de Pesaran & 0.583 & 1.663 \\
Teste de Friedman & $32.332^{* * *}$ & $36.222^{* * *}$ \\
Teste de Breusch-Pagan LM & $171.750^{* * *}$ & $173.220^{* * *}$
\end{tabular}


Teste de Wooldridge

$55.189 * * *$

$57.062 * * *$

Notas: $* * *, * * \mathrm{e}^{*}$ denotam significância de $1 \%, 5 \%$ e $10 \%$, respetivamente. Teste de $\mathrm{Wald}_{0}$ : sigma(i)^2 para todo o $\mathrm{i}$; teste de Pesaran/Friedman e Breusch-Pagan $\mathrm{LM} \mathrm{H}_{0}$ : resíduos não estão correlacionados; teste de Wooldridge $\mathrm{H}_{0}$ : não existe auto-correlação de primeira ordem.

Os resultados da Tabela 6 rejeitam a hipótese nula do teste de Wald Modificado, concluindo que existe heterocedasticidade dos resíduos. O teste Pesaran não é significante, porém o teste Friedman, semelhante ao Pesaran, mostra que há uma dependência transversal dos erros, há assim uma correlação contemporânea. Por fim, os testes Breusch-Pagan e Wooldridge demonstram que os resíduos estão correlacionados e que há autocorrelação de primeira ordem, respetivamente, em ambos os modelos estudados.

Após esta bateria de testes conclui-se que o melhor estimador para os nossos modelos é o Driscoll-Kray (1998) (e.g. Hoechle, 2007; Fuinhas et al., 2015). Este modelo é um estimador de matriz que gera o erro padrão robusto para várias ocorrências, entre elas os erros encontrados nas amostras. A Tabela 7 apresenta os resultados dos modelos com os estimadores de referência estudados.

Tabela 7. Estimação dos resultados

\begin{tabular}{|c|c|c|c|c|c|c|}
\hline \multicolumn{7}{|c|}{ Variável dependente: DLYPC } \\
\hline & \multicolumn{3}{|c|}{ Modelo 1} & \multicolumn{3}{|c|}{ Modelo 2} \\
\hline Modelos & $\mathrm{FE}(\mathrm{I})$ & FE rob $(\mathrm{I})$ & DK (I) & FE (II) & $\begin{array}{l}\text { FE rob } \\
\text { (II) }\end{array}$ & DK (II) \\
\hline Constante & $1.3628 * * *$ & $1.3628 * * *$ & $1.3628 * * *$ & $1.0577 * * *$ & $1.0577 * * *$ & $1.0577 * * *$ \\
\hline DSE & $0.0609 * * *$ & $0.0609 * * *$ & $0.0609 * * *$ & $0.0547 * * *$ & $0.0547 * * *$ & $0.0547 * * *$ \\
\hline DLORPC & $\begin{array}{c}- \\
0.0307 * * *\end{array}$ & $\begin{array}{c}- \\
0.0307 * * *\end{array}$ & $\begin{array}{c}- \\
0.0307 * * *\end{array}$ & $\begin{array}{c}- \\
0.0238^{*} * *\end{array}$ & $-0.0238 * *$ & $\begin{array}{c}- \\
0.0238^{* * *}\end{array}$ \\
\hline DLP & $0.0522 * * *$ & $0.0522 * * *$ & $0.0522 * * *$ & $0.0479 * * *$ & $0.0479 * * *$ & $0.0479 * * *$ \\
\hline DDF & $\begin{array}{c}- \\
0.0697 * * *\end{array}$ & -0.0697 & -0.0697 & $\begin{array}{c}- \\
0.0719 * * *\end{array}$ & -0.0719 & -0.0719 \\
\hline DLEXPC & $0.0242 * *$ & $0.0242 *$ & 0.0242 & $0.0206^{*}$ & 0.0206 & 0.0206 \\
\hline $\begin{array}{l}\text { DLCO2PC } \\
\text { DLOCPC }\end{array}$ & $0.3709 * * *$ & $0.3709 * * *$ & $0.3709 * * *$ & $0.3532 * * *$ & $0.3532 * * *$ & $0.3532 * * *$ \\
\hline LYPC (-1) & $\begin{array}{c}- \\
0.1258 * * *\end{array}$ & $\begin{array}{c}- \\
0.1258 * * *\end{array}$ & $\begin{array}{c}- \\
0.1258 * * *\end{array}$ & $\begin{array}{c}- \\
0.1039 * * *\end{array}$ & $\begin{array}{c}- \\
0.1039 * * *\end{array}$ & $\begin{array}{c}- \\
0.1039 * * *\end{array}$ \\
\hline $\mathrm{SE}(-1)$ & $0.0097 * *$ & 0.0097 & $0.0097 * *$ & 0.0021 & 0.0021 & 0.0021 \\
\hline $\begin{array}{l}\text { LORPC (- } \\
1)\end{array}$ & $\begin{array}{c}- \\
0.0166^{* * * *}\end{array}$ & $\begin{array}{c}- \\
0.0166^{*} * *\end{array}$ & $\begin{array}{c}- \\
0.0166^{* * *}\end{array}$ & $\begin{array}{c}- \\
0.0129 * * *\end{array}$ & $-0.0129 * *$ & $\begin{array}{c}- \\
0.0129 * * *\end{array}$ \\
\hline LP $(-1)$ & $0.0156^{* * *}$ & $0.0156^{* * *}$ & $0.0156^{* * *}$ & $0.0145 * * *$ & $0.0145 * *$ & $0.0145^{* *}$ \\
\hline DF $(-1)$ & -0.0097 & -0.0098 & -0.0099 & -0.0163 & -0.0164 & -0.0165 \\
\hline LEXPC (-1) & $0.0511 * * *$ & $0.0511 * * *$ & $0.0511 * * *$ & $0.0480 * * *$ & $0.0480 * * *$ & $0.0480 * * *$ \\
\hline
\end{tabular}




\begin{tabular}{|c|c|c|c|c|c|c|}
\hline $\begin{array}{l}\mathrm{LCO} 2 \mathrm{PC}(- \\
\text { 1) } \\
\text { LOCPC (- } \\
\text { 1) }\end{array}$ & $0.0380 * * *$ & $0.0380 * *$ & $0.0380 * * *$ & $0.0262 * *$ & 0.0262 & $0.0262 * *$ \\
\hline \multicolumn{7}{|l|}{ Estatísticas } \\
\hline $\mathrm{N}$ & 450 & 450 & 450 & 450 & 450 & 450 \\
\hline $\mathrm{R}^{2}$ & 0.5407 & 0.5407 & 0.5407 & 0.5146 & 0.5146 & 0.5146 \\
\hline $\mathrm{R}^{2} \_\mathrm{a}$ & 0.5113 & 0.5270 & & 0.4836 & 0.5002 & \\
\hline $\mathrm{F}$ & $38.22 * * *$ & & $131.76^{* * *}$ & $34.42 * * *$ & & $62.41 * * *$ \\
\hline
\end{tabular}

Notas: ***, $* * \mathrm{e}^{*}$ denotam significância de $1 \%, 5 \%$ e $10 \%$, respetivamente. Os comandos utilizados no software Stata foram xtreg e xtscc. Na estimação do DK foi utilizado 1 lag.

Após a estimação dos modelos FE, FE Robust (FE rob) e Driscoll-Kray (DK) procedemos ao cálculo das elasticidades e semi-elasticidades. De referir que as elasticidades de longo prazo não são de leitura direta como as de curto prazo (ver Tabela 8), pois as de curto prazo são iguais aos seus coeficientes. Assim, para o cálculo das elasticidades de longo prazo foi necessário dividir o coeficiente de cada variável explicativa pelo coeficiente da variável dependente (LYPC), desfasadas uma vez e multiplicadas por $(-1)$.

Tabela 8. Elasticidades e velocidade de ajustamento

\begin{tabular}{|c|c|c|c|c|c|c|}
\hline \multicolumn{7}{|c|}{ Variável dependente: DLYPC } \\
\hline & \multicolumn{3}{|c|}{ Modelo 1} & \multicolumn{3}{|c|}{ Modelo 2} \\
\hline Modelos & FE (I) & FE rob (I) & DK (I) & FE (II) & $\begin{array}{c}\text { FE rob } \\
\text { (II) }\end{array}$ & DK (II) \\
\hline \multicolumn{7}{|c|}{ Semi-elasticidades de curto prazo } \\
\hline DSE & $0.0609 * * *$ & $0.0609 * * *$ & $0.0609 * * *$ & $0.0547 * * *$ & $0.0547 * * *$ & $0.0547 * * *$ \\
\hline DLORPC & $\begin{array}{c}- \\
0.0307 * * *\end{array}$ & $\begin{array}{c}- \\
0.0307 * * *\end{array}$ & $\begin{array}{c}- \\
0.0307 * * *\end{array}$ & $0.0238 * * *$ & $-0.0238 * *$ & $\begin{array}{c}- \\
0.0238 * * *\end{array}$ \\
\hline DLP & $0.0522 * * *$ & $0.0522 * * *$ & $0.0522 * * *$ & $0.0479 * * *$ & $0.0479 * * *$ & $0.0479 * * *$ \\
\hline DDF & $\begin{array}{c}- \\
0.0697 * * *\end{array}$ & -0.0697 & -0.0697 & $\begin{array}{c}- \\
0.0719 * * *\end{array}$ & -0.0719 & -0.0719 \\
\hline DLEXPC & $0.0242 * *$ & $0.0242 *$ & 0.0242 & $0.0206 *$ & 0.0206 & 0.0206 \\
\hline $\mathrm{DLCO} 2 \mathrm{PC}$ & $0.3709 * * *$ & $0.3709 * * *$ & $0.3709 * * *$ & & & \\
\hline DLOCPC & & & & $0.3532 * * *$ & $0.3532 * * *$ & $0.3532 * * *$ \\
\hline \multicolumn{7}{|c|}{ Elasticidades de longo prazo } \\
\hline $\mathrm{SE}$ & $0.0768 * *$ & $0.0768 *$ & $0.0768 * *$ & 0.0202 & 0.0202 & 0.0202 \\
\hline LORPC & $\begin{array}{c}- \\
0.1317 * * *\end{array}$ & $\begin{array}{c}- \\
0.1317^{*} * *\end{array}$ & $\begin{array}{c}- \\
0.1317^{*} * *\end{array}$ & - & $-0.1252 * *$ & $\begin{array}{c}- \\
0.1252 * * *\end{array}$ \\
\hline LP & $0.1239 * * *$ & $0.1239 * * *$ & $0.1239 * * *$ & $0.1397 * * *$ & $0.1397 * *$ & $0.1397^{* *}$ \\
\hline DF & -0.0770 & -0.0771 & -0.0772 & -0.1566 & -0.1566 & -0.1566 \\
\hline LEXPC & $0.4058 * * *$ & $0.4058 * * *$ & $0.4058 * * *$ & $0.4630 * * *$ & $0.4630 * * *$ & $0.4630 * * *$ \\
\hline $\mathrm{LCO} 2 \mathrm{PC}$ & $0.3019 * * *$ & $0.3019 * *$ & $0.3019 * * *$ & & & \\
\hline LOCPC & & & & $0.2519 * *$ & $0.2519 * *$ & $0.2519 * *$ \\
\hline
\end{tabular}




\begin{tabular}{l|ccc|ccc}
\hline \multicolumn{7}{l}{ Velocidade de ajustamento } \\
\hline \multirow{2}{*}{ ECM } & - & - & - & - & - & - \\
& $0.1258 * * *$ & $0.1258 * * *$ & $0.1258 * * *$ & $0.1039 * * *$ & $0.1039 * * *$ & $0.1039 * * *$ \\
\hline
\end{tabular}

Notas: $* * *, * * \mathrm{e}^{*}$ denotam significância de $1 \%, 5 \%$ e $10 \%$, respetivamente. Os comandos utilizados no software Stata foram xtreg e xtscc. Na estimação do DK foi utilizado 1 lag.

Os resultados anteriores demonstram o efeito que as variáveis aplicadas no modelo têm sobre o CE. Estes determinantes impostos no modelo apresentam uma característica linear, nomeadamente a variável DF, e que não apresenta valores significantes.

\subsection{Relação não linear entre DF e CE}

Utilizando apenas a variável DF como proxy para o desenvolvimento financeiro, observamos apenas o impacto que esta variável tem de forma linear. Diversos autores (Easterly et al., 2000; Arcand et al., 2012) ao realizarem a sua pesquisa encontraram uma associação de não linearidade entre DF e CE. Também em países de alto e baixo rendimento, há uma interação não linear entre o nexus Desenvolvimento-Crescimento (Deidda e Fattouh, 2002). Além disso, Arcand et al. (2012) argumentam no seu estudo que se o modelo não permitir estudar a não linearidade entre as variáveis, este pode apresentar uma descendente e não perfeita estimação sobre o impacto do $\mathrm{DF}$ no $\mathrm{CE}$ e vice-versa.

Até à literatura mais recente, ainda não existe uma abordagem única para a modelação da não linearidade entre as variáveis que desejamos estudar. O método proposto por Lind e Mehlum (2010) testa a existência de uma forma em U ou um U invertido, confirmando a existência ou não de uma relação monótona entre DF e CE. Este teste tem como metodologia a junção de uma função polinomial quadrática. A estimação do modelo é a seguinte ${ }^{4}$, Eq.(5):

$$
L Y P C_{i}=a D F_{i}+b D F_{i}^{2}+Z_{i} C+\varepsilon_{i}
$$

onde a hipótese nula $\left(\mathrm{H}_{0}\right)$ é, Eq.(6):

$$
H_{o}:\left(a+b 2 \mathrm{DF}_{\min } \leq 0\right) \mathrm{U}\left(a+b 2 \mathrm{DF}_{\min } \geq 0\right)
$$

e a hipótese alternativa $\left(\mathrm{H}_{1}\right)$ é, Eq.(7):

\footnotetext{
${ }^{4}$ Segundo Lind e Mehlum (2010) Samargandi et al (2015).
} 


$$
H_{1}:\left(a+b 2 \mathrm{DF}_{\min }>0\right) \mathrm{U}\left(a+b 2 \mathrm{DF}_{\min }<0\right)
$$

Sendo que o DFmin e o DFmax representam o mínimo e o máximo que os valores do DF representam. Se a hipótese nula for rejeitada, confirma-se a existência de um U invertido (Tabela 9). O resultado do Teste $U$ é igual para ambos os modelos (1 e 2) propostos inicialmente.

\section{Tabela 9. Teste U}

Declive do DFmin

Declive do DFmax

SLM teste para o U invertido

P-valor

\section{$2.234 * *$}

$-5.155 * * *$

1.83

0.03

Notas: ***,** e $*$ denotam significância de $1 \%, 5 \%$ e $10 \%$

Pode-se verificar, através dos resultados do teste U, que os valores do limite superior (-5.155) e inferior (2.234) são significantes, o que significa a rejeição da hipótese nula (não há U invertido), logo a existência de uma relação não linear entre DF e CE é confirmada.

A Tabela 10 mostra as estimações dos modelos, as elasticidades e velocidades de ajustamento com a incorporação da variável DF ao quadrado ( $\left.\mathrm{DF}^{\wedge} 2=\mathrm{DFSQ}\right)$.

Tabela 10. Elasticidades e velocidade de ajustamento

\begin{tabular}{|c|c|c|c|c|c|c|}
\hline \multicolumn{7}{|c|}{ Variável dependente: DLYPC } \\
\hline & \multicolumn{3}{|c|}{ Modelo 1} & \multicolumn{3}{|c|}{ Modelo 2} \\
\hline Modelos & FE (I) & FE rob (I) & DK (I) & FE (II) & FE rob (II) & $\overline{\mathrm{DK}}(\mathrm{II})$ \\
\hline \multicolumn{7}{|c|}{ Semi-elasticidades de curto prazo } \\
\hline DSE & $0.0617 * * *$ & $0.0617 * * *$ & $0.0617 * * *$ & $0.0541 * * *$ & $0.0541 * * *$ & $0.0541 * * *$ \\
\hline DLORPC & $0.0301 * * *$ & $0.0301 * * *$ & $0.0301 * * *$ & $-0.0229 * * *$ & $-0.0229 * *$ & $0.0229 * * *$ \\
\hline DLP & $0.0512 * * *$ & $0.0512 * * *$ & $0.0512 * * *$ & $0.0462 * * *$ & $0.0462 * * *$ & $0.0462 * * *$ \\
\hline DPF & $0.1640 * *$ & $0.1640 * * *$ & $0.1640^{*}$ & 0.018 & 0.018 & 0.018 \\
\hline DPFSQ & $\frac{-}{0.1574 * * *}$ & $0.1574 * * *$ & $0.1574 * * *$ & $-0.1240 * * *$ & $-0.1240 * *$ & $-0.1240 * *$ \\
\hline DLEXPC & $0.0249 * *$ & $0.0249 *$ & 0.0249 & $0.0204 *$ & 0.0204 & 0.0204 \\
\hline DLCO2PC & $0.3516^{* * *}$ & $0.3516^{* * *}$ & $0.3516 * * *$ & & & \\
\hline DLOCPC & & & & $0.3309 * * *$ & $0.3309 * * *$ & $0.3309 * * *$ \\
\hline \multicolumn{7}{|c|}{ Elasticidades de longo prazo } \\
\hline SE & $0.0839 * *$ & $0.0839 *$ & $0.0839 * *$ & 0.0182 & 0.0182 & 0.0182 \\
\hline LORPC & $\begin{array}{c}- \\
0.1207 * * *\end{array}$ & $\begin{array}{c}- \\
0.1207 * * *\end{array}$ & $\begin{array}{c}- \\
0.1207 * * *\end{array}$ & $-0.1117 * * *$ & $-0.1117 *$ & $\begin{array}{c}- \\
0.1117^{* * *}\end{array}$ \\
\hline
\end{tabular}




\begin{tabular}{l|ccc|ccc} 
LP & $0.1212 * * *$ & $0.1212 * * *$ & $0.1212 * * *$ & $0.1315^{* * *}$ & $0.1315^{* *}$ & $0.1315^{* *}$ \\
DF & $0.4948^{* *}$ & $0.4948^{* *}$ & $0.4948^{*}$ & $0.5319^{*}$ & 0.5319 & 0.5319 \\
DFSQ & $0.4406^{* * *}$ & $0.4406^{* * *}$ & $0.4406^{* * *}$ & $-0.5275^{* *}$ & $-0.5275^{* *}$ & $-0.5275^{* *}$ \\
LEXPC & $0.3873^{* * *}$ & $0.3873 * * *$ & $0.3873^{* * *}$ & $0.4675^{* * *}$ & $0.4675 * * *$ & $0.4675^{* * *}$ \\
LCO2PC & $0.3421 * * *$ & $0.3421 * * *$ & $0.3421 * * *$ & & & \\
LOCPC & & & & $0.2245 *$ & 0.2245 & $0.2245 *$ \\
\hline
\end{tabular}

Velocidade de ajustamento

\begin{tabular}{l|ccc|ccc}
\hline \multirow{2}{*}{$\mathrm{ECM}$} & - & - & - & \multirow{2}{*}{$0.0999 * * *$} & - & - \\
& $0.1264 * * *$ & $0.1264 * * *$ & $0.1264 * * *$ & & $0.0999 * * *$ & $0.0999 * * *$ \\
\hline
\end{tabular}

Notas: $* * *, * *$ e $*$ denotam significância de $1 \%, 5 \%$ e $10 \%$, respetivamente. Os comandos utilizados no software Stata foram xtreg e xtscc. Na estimação do DK foi utilizado 1 lag.

Pode-se observar que as elasticidades, em relação à Tabela 8, alteram os valores e os níveis de significância estatística de algumas variáveis, em destaque a variável DF. Estes resultados vão ao encontro da literatura existente que revela que o CE só é afetado positivamente pelo DF até um determinado ponto e depois essa relação torna-se negativa (e.g., Arcand et al., 2012; Samargandi et al., 2015).

Para verificar a robustez do nosso estudo relativo à interação desenvolvimentocrescimento, criámos uma nova base de dados, com menos países (11) e com um horizonte temporal mais longo (1972-2011). A redução de países ocorre devido à falta de dados para os 4 países (Brasil, Argélia, Indonésia, Tailândia) excluídos. Este complementar da análise relaciona-se com o fato de o DF perder força sobre o CE com o passar do tempo, tornando-se negativo a partir de um determinado ponto (Rousseau e Wachtel, 2011).

A Tabela 11 mostra os resultados das elasticidades e das velocidades de ajustamento dos modelos com um horizonte temporal mais longo (Modelo 1A e Modelo $\left.2^{\mathrm{a}}\right)$.

Tabela 11. Elasticidades e velocidade de ajustamento

\begin{tabular}{l|ccc|ccc}
\hline \multicolumn{7}{l}{ Variável dependente: DLYPC } \\
\hline \multicolumn{7}{c}{ Modelo 1A } \\
\hline Modelos & FE (I) & FE rob (I) & DK (I) & FE (II) & FE rob (II) & DK (II) \\
\hline \multicolumn{1}{l}{ Semi-elasticidades de curto prazo } \\
\hline DSE & $0.0407 * * *$ & $0.0407 * * *$ & $0.0407 * * *$ & $0.0399^{* * *}$ & $0.0399^{* * *}$ & $0.0399^{* * *}$ \\
DLORPC & - & $-0.0294 *$ & $-0.0294 * *$ & $-0.0289 * * *$ & - & - \\
DLP & $0.0294^{* * *}$ & $-0.0289^{* * *}$ & $0.0289 * * *$ \\
DPF & $0.0383^{* * *}$ & $0.0383^{* * *}$ & $0.0383^{* * *}$ & $0.0379 * * *$ & $0.0379 * *$ & $0.0379 * * *$ \\
DPFSQ & -0.0096 & -0.0096 & -0.0096 & -0.0759 & -0.0759 & -0.0759 \\
\hline \hline
\end{tabular}




\begin{tabular}{|c|c|c|c|c|c|c|}
\hline $\begin{array}{l}\text { DLEXPC } \\
\text { DLCO2PC } \\
\text { DLOCPC }\end{array}$ & $\begin{array}{c}0.0266^{* *} \\
0.3029^{* * *}\end{array}$ & $\begin{array}{c}0.0266^{* *} \\
0.3029^{* * *}\end{array}$ & $\begin{array}{c}0.0266 \\
0.3029 * * *\end{array}$ & $\begin{array}{l}0.0327 * * * \\
0.2939 * * *\end{array}$ & $\begin{array}{l}0.0327 * * * \\
0.2939 * * *\end{array}$ & $0.2939 * * *$ \\
\hline \multicolumn{7}{|c|}{ Elasticidades de longo prazo } \\
\hline$\overline{\mathrm{SE}}$ & $0.0903 * * *$ & $0.0903 * * *$ & $0.0903 * * *$ & $0.0828 * * *$ & $0.0828 * * *$ & $0.0828 * * *$ \\
\hline LORPC & $-0.0605^{* *}$ & -0.0605 & $\stackrel{-}{0.0605 * * *}$ & $-0.0838 * * *$ & $-0.0838^{*}$ & $\begin{array}{c}- \\
0.0838 * * *\end{array}$ \\
\hline LP & 0.0322 & 0.0322 & 0.0322 & $0.0719 *$ & 0.0719 & $0.0719^{*}$ \\
\hline DF & $1.9596^{* * *}$ & $1.9596 * * *$ & $1.9596^{* * *}$ & $1.8962 * * *$ & $1.8962 * *$ & $1.8962 * * *$ \\
\hline DFSQ & $\begin{array}{c}- \\
1.2649 * * *\end{array}$ & $1.2649 * * *$ & $\begin{array}{c}- \\
1.2649 * * *\end{array}$ & $-1.2145^{* * *}$ & $-1.2145^{* *}$ & $1.2145^{-} * * *$ \\
\hline LEXPC & $0.4099 * * *$ & $0.4099 * * *$ & $0.4099 * * *$ & $0.5048 * * *$ & $0.5048 * * *$ & $0.5048 * * *$ \\
\hline $\mathrm{LCO} 2 \mathrm{PC}$ & $0.2729 * * *$ & $0.2729 * *$ & $0.2729 * *$ & & & \\
\hline LOCPC & & & & $0.1553 *$ & 0.16 & 0.16 \\
\hline \multicolumn{7}{|c|}{ Velocidade de ajustamento } \\
\hline ECM & $\begin{array}{c}- \\
0.0954 * * *\end{array}$ & $\begin{array}{c}- \\
0.0954 * * *\end{array}$ & $\begin{array}{c}- \\
0.0954 * * *\end{array}$ & $-0.0815 * * *$ & $-0.0815^{* *}$ & $\begin{array}{c}- \\
0.0815^{* * *}\end{array}$ \\
\hline
\end{tabular}

Observa-se a partir das Tabelas 10 e 11 que no curto prazo as variáveis rácio de produção de petróleo sobre energia primária (SE), rendas de petróleo (ORPC), preços de petróleo $(\mathrm{P})$, emissões de $\mathrm{CO}_{2}(\mathrm{CO} 2 \mathrm{PC})$ e consumo de petróleo (OCPC) são estatisticamente significantes nos quatro modelos apresentados. Apenas o Modelo 1 apresenta a existência de um $U$ invertido do DF no curto prazo. No longo prazo os coeficientes alteram-se e os níveis de significância estatística tornam-se diferentes. As variáveis que apresentam coeficientes relativamente diferentes são as DF e DFSQ. Pode-se afirmar que no curto prazo e longo prazo, apenas o Modelo 1 (Tabela 10) com as emissões de $\mathrm{CO}_{2}$ apresenta uma relação não linear entre DF e CE. Nos modelos $1 \mathrm{~A}$ e 2A (Tabela 11) os resultados mantêm-se apenas no longo prazo, embora os níveis de significância se alterem para 1\% nas variáveis financeiras (DF e DFSQ). As velocidades de ajustamento (ECM - mecanismo de correção dos erros) são estatisticamente altamente significantes para todos os estimadores (FE, Fe Robust e DK), provando que a recuperação das economias destes países a choques econômicos é lenta. 


\section{DISCUSSÃO}

Segundo o que foi mencionado nas seções anteriores, proceder-se-á à discussão dos resultados alcançados ao longo desta pesquisa. O foco principal deste estudo é o estudo da não linearidade com recurso a um painel de países produtores petróleo, com base em dados maioritariamente per capita. Através dos testes iniciais, comprova-se a existência de heterocedasticidade, a dependência transversal dos erros, a correlação dos resíduos, a existência de autocorrelação de primeira ordem, tal como todas as variáveis serem $\mathrm{I}(1)$ e $\mathrm{I}(0)$, logo exibem memória de longo prazo, em todos os modelos. A nossa análise está focada nos resultados com as variáveis DF e DFSF.

O consumo de petróleo (OCPC) a curto prazo, apresenta nos dois modelos (2 e 2A) uma significância positiva perante o PIB, contribuindo assim para o CE. No longo prazo a variável apenas apresentava valores estatisticamente significantes no modelo mais reduzido de tempo (2).

Como enunciado na revisão da literatura, as emissões de CO2 têm um impacto positivo quer no curto, quer no longo prazo. A análise empírica, em todos os modelos $(1,2,1 \mathrm{~A}$ e $2 \mathrm{~A})$, demonstra que as emissões de $\mathrm{CO}_{2}$ afetam positivamente o $\mathrm{CE}$, quer no curto, quer no longo prazo. Estes resultados estão em concordância com a secção teórica do artigo, dado que o consumo de petróleo e de energia aumenta as emissões de $\mathrm{CO}_{2}$ (e.g., Al-Mulali e Sab, 2012; Boutabba, 2014).

A proporção da produção de petróleo sobre o consumo de energia primária (SE) releva poder explicativo, tanto a curto como a longo prazo para 3 modelos (1, 1A e 2). $\mathrm{Na}$ análise das elasticidades da variável SE no modelo OLS verifica-se que o sinal do coeficiente é negativo e estatisticamente significante e com FE, FE rob e DK o sinal torna-se positivo e igualmente significante, demonstrando e reforçando a importância dos efeitos fixos na análise. Este fato decorre de as economias destes países apresentarem sintomas de dependência de petróleo e prenunciarem a existência de diferenças nas suas economias.

Em relação aos preços de petróleo $(\mathrm{P})$, quanto mais elevado for o preço, maior o rendimento dos países, logo perante estes acontecimentos, as elasticidades são positivas e significantes no curto prazo e no longo prazo apenas nos modelos com mais países (1 e 2), promovendo o CE. Nos modelos com maior período temporal (1A e 2A), os preços de petróleo apresentam menor significância estatística ou mesmo nula. Este aumento/diminuição dos preços de petróleo pode influenciar a estabilidade económica 
destes países. Assim as autoridades energéticas de cada país devem focar-se em aproveitar estes ganhos com as oscilações dos preços de petróleo para implementar pesquisas em outras energias, preferencialmente renováveis.

Como foi dito anteriormente, as rendas de petróleo são a diferença entre o valor de produção de petróleo bruto a preços mundiais subtraído dos seus custos de produção. Esta variável torna-se assim contrária ao $\mathrm{CE}$ em economias exportadoras de petróleo, já que um aumento dos custos de produção reduzirá o rendimento dos produtores. Desta forma a variável rendas de petróleo (ORPC) é negativa e estatisticamente significante, quer a curto, quer a longo prazo em todos as regressões realizadas.

Quanto às exportações de bens e serviços (EXPC), pode-se chegar duas conclusões: (i) no longo prazo, em todos os modelos as exportações são positivas e estatisticamente significantes, o que era expectável; (ii) no curto prazo o coeficiente apenas apresenta significância estatística quando o LOCPC é incluído nas regressões e com maior período temporal (Modelo 2A). Este resultado indica a possibilidade de haver uma maldição de recursos (resource curse), sendo que os decisores políticos dos países em análise deveriam procurar diversificar as suas economias (Lederman e Maloney, 2007).

Por último, as variáveis correspondentes ao DF demonstram resultados semelhantes. Na primeira análise (não linear) efetuou-se o estudo com uma proxy para o desenvolvimento do setor financeiro através do determinante M3, onde os resultados não permitiram chegar a valores estatisticamente significantes, concluindo que nesta amostra não há uma relação linear significante entre DF e CE. Todavia optou-se por uma abordagem distinta com base em métodos existentes na literatura (e.g. Rousseau e Wachtel, 2011; Arcand et al., 2012; Samargandi et al., 2015). Recorreu-se ao Teste U para verificar a não linearidade da estrutura em estudo, e incluímos a variável DFSQ nas regressões de modo a perceber qual o impacto que o DF tem no CE e aferir se este se comporta da mesma maneira ao longo do tempo. Conclui-se que nos modelos iniciais (1 e 2), apenas o primeiro, com as emissões de $\mathrm{CO}_{2}$, estabelece uma relação não linear entre DF e CE, no curto e no longo prazo. Embora os níveis de significância sejam iguais no curto e no longo prazo do primeiro modelo (1), a variável DF, no longo prazo, está mais próxima de apresentar um significância de 5\% do que 10\% (P-valor $=0.052$ ). Ou seja, o setor financeiro promove o $\mathrm{CE}$ da economia até certo ponto, sendo que essa influência se torna negativa com a evolução do setor financeiro. Pode-se dizer que no longo prazo o DF tem mais impacto sobre o CE. 
Para apurar a permanência e robustez da relação entre DF e CE, efetuou-se uma extensão do período temporal em 9 anos e a consequente extração de 4 países. De referir que os maiores produtores de petróleo permaneceram na amostra. Esta nova análise veio fomentar o nosso estudo, pois ao aumentarmos o horizonte temporal, os níveis de significância no curto prazo tornaram-se inexistentes e no longo prazo aumentaram para $1 \%$, em ambos os modelos (1A e 2A). As ilações retiradas vêm ajudar e complementar a literatura existente, provando que o setor financeiro ajudou o $\mathrm{CE}$ numa altura em que as instituições financeiras obtiveram impacto positivo e com o passar do tempo e consequente evolução dos mercados financeiros e do setor bancário, o DF atenuou, passando a influenciar negativamente o CE. Deve ter-se em conta que as instituições financeiras em economias exportadoras de petróleo são mais fracas em comparação com as economias importadoras desse mesmo bem (Nili e Rastad, 2007; Al-Malkawi et al., 2012).

As velocidades de ajustamento dos modelos são negativas e baixas $(8.15 \%<$ ECM < 12.64) nos quatro modelos, mas estatisticamente altamente significantes. Indiciando assim a limitação das economias presentes no estudo, por demorarem muito tempo a ultrapassar de eventuais choques econômicos. Este problema não se deve apenas à alta dependência de apenas alguns setores, mas também pela apatia existente nesses setores, altamente dependentes, afetando a economia global dos países. O ECM demonstra que no longo prazo todas as variáveis são responsáveis por alterações existentes no CE.

Com estas conclusões e sabendo que as variáveis $\mathrm{LOCPC}$ e $\mathrm{LCO}_{2} \mathrm{PC}$ são colineares, os dados empíricos revelam que as emissões de $\mathrm{CO}_{2}$ apresentam resultados mais robustos e com mais impacto do que com o consumo de petróleo.

Os resultados alcançados reforçam e são consistentes com a literatura existente (e.g. Rousseau e Wachtel, 2011; Arcand et al., 2012; Samargandi et al., 2015). Além disso permitem complementar a investigação empírica, já que poucos estudos relacionam $\mathrm{DF}, \mathrm{CE}$, países produtores de petróleo e emissões de $\mathrm{CO}_{2}$, com as técnicas econométricas utilizadas no estudo e de uma forma não linear.

\section{Conclusão}

O nexus desenvolvimento financeiro - crescimento econômico em países produtores de petróleo é analisado no artigo, onde incluímos variáveis relacionadas com 
o meio ambiente, como emissões de $\mathrm{CO}_{2}$, consumo de petróleo, rácio produção de petróleo sobre energia primária, rendas e preços de petróleo e variáveis financeiras, como M3. O estudo está focado num painel inicial de 15 países com um período temporal de 1981 a 2011. Posteriormente a amostra foi reduzida para 11 países, resultando num aumento do horizonte temporal para 1972-2011. Os resultados empíricos encontrados complementam a literatura existente, sendo que as exportações têm mais impacto no longo do que no curto prazo em ambos os modelos estudados. O consumo de petróleo é estatisticamente altamente significante nos quatro estudos de modelos realizados mas apenas no curto prazo, já o mesmo não acontece com as rendas de petróleo, que são negativas e significantes nas suas elasticidades e semielasticidades. Os preços de petróleo produzem riqueza às economias exportadoras de energia fóssil, quer no curto como no longo prazo. O consumo de petróleo faz com que aumente as emissões de $\mathrm{CO}_{2}$, este facto pode ser comprovado pelos valores positivos e estatisticamente altamente significantes, contribuindo para a criação de riqueza. As exportações de bens e serviços apenas apresentam resultados estatisticamente significantes no longo prazo.

Em relação à proxy de Desenvolvimento Financeiro (M3/PIB), pode-se aferir que a variável não apresenta valores estatisticamente significantes em termos lineares, provando que não há uma relação linear entre DF e CE. Com estes resultados e com o que é evidenciado na literatura recorreu-se a um método para aferir a não linearidade e se o efeito do DF sobre o CE é positivo ou negativo. Demonstra-se a existência de um U invertido, em ambos os modelos, com 11 e com 15 países. Com este método, encontraram-se resultados que demonstram que no longo prazo há uma relação não linear entre DF e CE. No curto prazo apenas há uma relação no Modelo 1, onde as emissões de $\mathrm{CO}_{2}$ são incluídas, e com níveis de significância baixos.

Concluindo, o DF tem vantagens e desvantagens perante o CE. Tem um impacto positivo quando o setor financeiro não é evoluído, como o rápido crescimento do crédito nos mercados em economias pouco desenvolvidas, originando um aumento do PIB. O impacto negativo poderá estar associado às crises que afetam o setor financeiro, à fragilização do setor bancário e ao nível de financiamento realizados pelos bancos nas economias em análise.

Estes resultados para além de complementarem a literatura teórica e empírica, contribuem no sentido de aprofundar a não linearidade do DF perante o $\mathrm{CE}$ em economias exportadoras de petróleo. A inclusão de determinantes energéticos na análise 
do tema é uma inovação na área em estudo. Provámos que as instituições financeiras de países exportadores de petróleo são fracas e que o setor financeiro ou ainda não está suficientemente desenvolvido em determinados países (e.g. Egito, Argélia) ou esse desenvolvimento já não influência positivamente o setor financeiro em determinadas economias de alto rendimento da análise (e.g. Itália, Estados Unido da América).

Para futuras pesquisas, recomenda-se o alargamento do período temporal e o número de países passíveis de ser incorporados. A utilização da Análise dos Componentes Principais é recomendada para constituir um índice, de forma a agregar diversas variáveis financeiras e melhorar a proxy para Desenvolvimento Financeiro.

\section{Referências}

Al-Malkawi, H., Marashdeh, H., Abdullah, N. (2012). Financial Development and Economic Growth in the UAE: Empirical Assessment Using ARDL Approach to Co-integration. Internacional Journal of Economics and Finance, 4(5).

Al-Mulali, U., Sab, C.N.B.C. (2012). The impact of energy consumption and CO2 emissions on the economic growth and financial development in the Sub Saharan African countries. Energy, 39(1), 180-186.

Ang, J.B., e McKibbin, W.J. (2007). Financial liberalization, financial sector development and growth: Evidence from Malaysia. Journal of Development Economics, 84(1), 215-233.

Apergis, N., Payne, J.E. (2009). Energy consumption and economic growth in Central America: Evidence from a panel cointegration and error correction model. Energy Economics, 31(2), 211-216.

Arcand, J., Berkes, E., Panizza, U. (2012). Too much finance? International Monetary Fund, Research Department.

Baltagi, B.H. (2005). Econometric Analysis of Panel Data. Third edition, John Wiley and Sons.

Barro, R.J. (1991). Economic growth in a cross section of countries. Quarterly Journal of Economics, 106(2), 407-443.

Beck, T. (2008). The econometrics of finance and growth. In Palgrave handbook of econometrics, (2), Palgrave Macmillan.

Beck, T., Levine, R., Loayza, N. (2000). Finance and the sources of growth. Journal of Financial Economics, 58(1), 261-300.

Birdsall, N., Wheeler, D. (1993). Trade policy and industrial pollution in Latin America: where are the pollution havens? Journal of Environment and Development, 2(1), 137-149.

Boutabba, M.A. (2014). The impact of financial development, income, energy and trade on carbon emissions: Evidence from Indian economy. Economic Modelling, 40, $33-41$.

Breitung, J. (2000). The local power of some unit root tests for panel data. Advances in Econometrics, 15, 161-177.

Calderon, C., Liu, L. (2003). The direction of causality between financial development and economic growth. Journal of Development Economics, 72(1), 321-334. 
Cecchetti, G., Kharroubi, E. (2012). Reassessing the impact of finance on growth. BIS Working, Bank for International Settlements, 381.

Choi, I. (2001). Unit root tests for panel data. Journal of International Money and Finance, 20(1), 249-272.

Christopoulos, D.K., Tsionas, E.G. (2004). Financial development and economic growth: Evidence from panel unit root and cointegration tests. Journal of Development Economics, 73(1), 55-74.

Chuah. H.L., Thai, W. (2004). Financial development and economic growth: Evidence from causality tests for the GCC countries. IMF Working Paper No. 04/XX.

Ciarlone, A. (2011). Housing wealth effect in emerging economies. Emerging Markets Review, 12(4), 399-417.

Claessens, S., Feijen, E. (2007). Financial sector development and the millennium development goals. World Bank Working Paper, The World Bank, 89.

Çoban, S., Topcu, M., (2013). The nexus between financial development and energy consumption in the EU: A dynamic panel data analysis. Energy Economics, 39, 81-88.

Dawson, P.J. (2010). Financial development and economic growth: A panel approach. Applied Economics Letters, 17(8), 741-745.

Deidda, L., Fattouh, B. (2002). Non-linearity between finance and growth. Economics Letters, 74(3), 339-345.

Demetriades, P.O., Hussein, K.A. (1996). Does financial development cause economic growth? Time-series evidence from 16 countries. Journal of Development Economics, 51(2), 387-412.

Dincecco, M. (2010). The political economy of fiscal prudence in historical perspective. Economics and Politics, 22(1), 1-36.

Dormann, C.F., Elith, J., Bacher, S., Buchmann, C., Carl, G., Carré, G., Marquéz, J.R.G., Gruber, B., Lafourcade, B., Leitão, P.J., Münkemüller, T., Mcclean, C., Osborne, P.E., Reineking, B., Schröder, B., Skidmore, A.K., Zurell, D., Lautenbach, S. (2013). Collinearity: A review of methods to deal with it and a simulation study evaluating their performance. Ecography, 36(1), 27-46.

Driscoll, J., Kraay, A.C. (1998). Consistent covariance matrix estimation with spatially dependent data. Review of Economics and Statistics, 80(4), 549-560.

Easterly, W., Islam, R., Stiglitz, J.E. (2000). Explaining growth volatility. The World Bank, mimeo.

Eberhardt, M. (2011). Panel time-series modeling: New tools for analyzing xt data. 2011 UK Stata Users Group meeting.

Favara, G. (2003). An empirical reassessment of the relationship between finance and growth. IMF Working Paper No. 03/123.

Frankel, J., Romer, D. (1999). Does trade cause growth? The American Economic Review, 89(3), 379-399.

Frankel, J., Rose, A. (2002). An estimate of the effect of common currencies on trade and income. Quarterly Journal of Economics, 117(2), 437-466.

Fuinhas, J. A., Marques, A.C., Couto, A P. (2015). Oil-Growth nexus in Oil Producing Countries: Macro Panel Evidence. Internacional Journal of Energy Economics and Policy, 5(1), 148-163.

Goldsmith R.W. (1969). Financial structure and development. New York: Yale University Press.

Graff, M. (2002). Casual Links Between Financial Activity and Economic Growth: Empirical Evidence from a Cross-Country Analysis, 1970-1990. Bulletin of Economic Research, 54(2), 110-133. 
Green, W. (2008). Econometrics Analysis. 6th ed., Prentice Hall.

Grossman, M., Krueger, A. (1995). Economic Growth and the Environment. The Quaterly Journal of Economics, 110(2), 353-377.

Gurley, J.G., Shaw, E.S. (1955). Financial aspects of economic development. The American Economic Review, 45(4), 515-538.

Hausman, J., Mcfaden, D. (1984). Specification test for multinominal logit model. Econometrica, 52, 1219-1240.

Hoechle, D. (2007). Robust standard errors for panel regressions with cross-sectional dependence. Stata Journal, 3, 281-312.

Hsiao, C. (2003). Analysis of panel data, second edition. Cambridge University. Cambridge, UK.

Im, K., Pesaran, H., Shin, Y. (2003). Testing for unit roots in heterogeneous panels. Journal of Econometrics, 115, 53-74.

Jalil A., Feridun, M. (2011). The impact of growth, energy and financial development on the environment in China: a cointegration analysis. Energy Economics, 33(2), 284-291.

Jedidia, K., Boujelbène, T., Helali, K. (2014). Financial development and economic growth: New evidence from Tunisia. Journal of Policy Modeling, 36(5), 883898.

Jensen, V. (1996). The pollution haven hypothesis and the industrial flight hypothesis: some perspectives on theory and empirics. Working Paper, 5, Centre for Development and the Environment, University of Oslo.

Kaminsky, G.L., Reinhart, C.M. (1999). The twin crises: the causes of banking and balance-of payments problems. The American Economic Review, 89(03), 473500.

Kar, M., Nazlioglu, S., Agir, H. (2011). Financial development and economic growth nexus in the MENA countries: Bootstrap panel granger causality analysis. Economic Modelling, 28(1), 685-693.

Keynes, J.M. (1988). A Teoria Geral do Emprego, do Juro e da Moeda. São Paulo: Nova Cultural.

Khan, M.S., Senhadji, A.S. (2003). Financial development and economic growth: A review and new evidence. Journal of African Economies, 12(2), 89-110.

King, R.G., Levine, R. (1993a). Finance, entrepreneurship, and growth: Theory and evidence. Journal of Monetary Economics, 32(3), 513-542.

King, R.G., Levine, R. (1993b). Finance and growth: Schumpeter might be right. The Quarterly Journal of Economics, 108(3), 717-737.

Klevmarken, N.A. (1989). Panel studies: what can we learn from them? Introduction. European Economic Review, 33, 523-529.

Kraft, J., Kraft, A. (1978). On the Relationship between Energy and GNP. Journal of Energy, 3, 401-403.

Krugman, P. (1996). Cycles of Conventional Wisdom on Economic Development. International Affair, 72.

Lean, H.H., Smith, R. (2009). CO $\mathrm{CO}_{2}$ emissions, energy consumption and output in ASEAN. Business and Economics, Development Research Unit Discussion Paper DEVDP 09-13.

Lederman, D., Maloney, W., (2007). Trade structure and growth. Natural Resources: Neither Curse Nor Destiny. Stanford Universtiy Press.

Levin, A., Lin, C.-F., Chu, C.-S.J. (2002). Unit root test in panel data: Asymptotic and finite-sample properties. Journal of Econometrics, 108(1), 1-24. 
Levine, R., Zervos, S. (1998). Stock markets, banks, and economic growth. The American Economic Review, 88(3), 537-558.

Lind, J.T., Mehlum, H. (2010). With or without U? The appropriate test for a U-shaped relationship. Oxford Bulletin of Economics and Statistics, 72(1), 109-118.

Loayza, N.V., Rancière, R. (2006). Financial development, financial fragility, and growth. Journal of Money, Credit and Banking, 38(4), 1051-1076.

Luintel, K.B., Khan, M. (1999). A quantitative reassessment of the finance-growth nexus: Evidence from a multivariate VAR. Journal of Development Economics, 60(2), 381-405.

Maddala, G.S., Wu, S. (1999). A comparative study of unit root tests with panel data a new simple test. Oxford Bulletin of Economics and Statistics, 61, 631-652.

McKinnon, R.I. (1973). Money and capital in economic development. Washington: Brookings Institution Press.

Minier, J. (2009). Opening a stock exchange. Journal of Development Economics, 90, $135-143$.

Narayan, P.K., Smyth, R. (2008). Energy Consumption and Real GDP in G7 Countries: New Evidence from Panel Cointegration with Structural Breaks. Energy Economics, 30(5), 2331-2341.

Nili, M., Rastad, M. (2007). Addressing the growth failure of the oil economies: The role of financial development. The Quaterly Review of Economics and Finance, 46, 726-740.

Ozturk, I. (2010). A literature survey on energy-growth nexus. Energy Policy, 38(1), 340-349.

Pesaran, M.H. (2007). A simple panel unit root test in the presence of cross section dependence. Journal of Applied Econometrics, 22(2), 265-312.

Pesaran, M.H., Shin, Y., Smith, R.P. (1999). Pooled Mean Group Estimation of Dynamic Heterogeneous Panels. Journal of American Statistical Association, 94(446), 621-634.

Roodman, D. (2006). How to do xtabond2: An introduction to difference and system GMM in stata. Center for Global Development Working Paper, 103.

Rousseau, P.L., Wachtel, P. (2011). What is happening to the impact of financial deepening on economic growth? Economic Inquiry, 49(1), 276-288.

Sadorsky, P. (2010). The impact of financial development on energy consumption in emerging economies. Energy Policy, 38, 2528-2535.

Sadorsky, P. (2011). Financial development and energy consumption in Central and Eastern European frontier economies. Energy Policy, 39, 9991006.

Saidi, K., Hammani, S. (2015). The impact of CO2 emissions and economic growth on energy consumption in 58 countries. Energy Reports, 1, 62-70.

Samargandi, N., Fidrmuc, J., Ghosh, S. (2014). Financial development and economic growth in an oil-rich economy: The case of Saudi Arabia. Economic Modelling, $43,267-278$.

Samargandi, N., Fidrmuc, J., Ghosh, S. (2015). Is the Relationship Between Financial Development and Economic Growth Monotonic? Evidence from a Sample of Middle-Income Countries. World Development, 68, 66-81.

Schumpeter, J.A., Opie, R. (1934). The theory of economic development: An inquiry into profits, capital, credit, interest, and the business cycle. Cambridge, Mass.: Harvard University Press.

Shahbaz, M., Solarin, A., Mahmood, H., Mohamed, A. (2013). Does financial development reduce $\mathrm{CO}_{2}$ emissions in Malaysian economy? A time series analysis. Economic Modelling, 35, 145-152. 
Shaw, E. (1973). Financial Deepening in Economic Development. New York: Oxford University Press.

Tamazian, A., Bhaskara Rao, B., (2010). Do economic, financial and institutional developments matter for environmental degradation? Evidence from transitional economies. Energy Economics, 32, 137-145.

Tamazian, A., Chousa, J.P., Vadlamannati, K.C. (2009). Does higher economic and financial development lead to environmmental degradation: Evidence from BRIC countries. Energy Policy, 37, 246-253.

Wachtel, P. (2003). How Much Do We Really Know about Growth and Finance? Economic Review, 88, 33-48.

Westerlund, J. (2007). Testing for error correction in panel data. Oxford Economics and Statistics, 69(6), 709-748.

Wolde-Rufael, Y. (2009). Energy Consumption and Economic Growth: The Experience of African Countries Revisited. Energy Economics, 31(2), 217-224.

World Bank (2000). Is globalization causing a 'race to the bottom' in environmental standard? PREM economic policy group and development economics group.

$\mathrm{Xu}, \mathrm{Z}$. (2000). Financial development, investment, and economic growth. Economic Inquiry, 38(2), 331-344.

Yuan, C., Liu, S., Xie, N. (2010). The Impact of Chinese Economic Growth and Energy Consumption of the Global Financial Crisis: An Input-Output Analysis. Energy 35(4): 1805-1812.

Zhang, X., Cheng, X. (2009). Energy Consumption, Carbon Emissions and Economic Growth in China. Ecological Economics, 68(10), 2706-2712.

Ziaei, S.M. (2015). Effects of financial development indicators on energy consumption and CO2 emissions of European, East Asian and Oceania countries. Renewable and Sustainable Energy Reviews, 42, 752-759.

Submetido em: 07/01/2016

Aceito em: 02/06/16 